\title{
MIMO Sliding Mode Control for a Tailless Fighter Aircraft, An Alternative to Reconfigurable Architectures
}

\author{
S. R. Wells ${ }^{1}$ and R. A. Hess ${ }^{2}$ \\ Dept. of Mechanical and Aeronautical Engineering \\ University of California \\ Davis, CA 95616-5294
}

\begin{abstract}
A frequency-domain procedure for the design of sliding mode controllers for multi-input, multi-output (MIMO) systems is presented. The methodology accommodates the effects of parasitic dynamics such as those introduced by unmodeled actuators through the introduction of multiple asymptotic observers and model reference hedging. The design procedure includes a frequency domain approach to specify the sliding manifold, the observer eigenvalues, and the hedge model. The procedure is applied to the development of a flight control system for a linear model of the Innovative Control Effector (ICE) fighter aircraft. The stability and performance robustness of the resulting design is demonstrated through the introduction of significant degradation in the control effector actuators and variation in vehicle dynamics.
\end{abstract}

\section{Introduction}

A reconfigurable control system is one which is able to compensate for sudden, potentially large, unknown failure eyents in real-time using on-line adaptive control laws and/or adaptive redistribution of control effort. The objective of reconfiguration is system stability while retaining some level of required performance and handling qualities. As a research area, reconfigurable flight control has seen rapid growth in the past decade. The motivation for developing reconfigurable flight controls is clear. Failures during flight are inevitable, especially in combat aircraft. If the flight control system is capable of stabilizing the aircraft and providing

\footnotetext{
${ }^{1}$ Major, USAF, Graduate Student, Student Member, AIAA

${ }^{2}$ Professor and Vice Chairman, Associate Fellow, AIAA
} 
acceptable handling qualities (if the aircraft is piloted), it may be possible to save the airframe and save lives. Many reconfigurable algorithms that have been discussed in the literature involve, to some degree, (1) failure detection, (2) system identification, and (3) flight control law reconfiguration. ${ }^{1-7}$ The time necessary to complete these operations can be critical, especially if the airframe in question is open-loop unstable. Even direct adaptive methods like dynamic inversion with neural nets to remove inversion error ${ }^{8-12}$ require some convergence time for adaptation. The method of sliding mode control (SMC) offers a characteristic which makes it very attractive for a reconfigurable application. The variable structure nature of the SMC controller allows it to adapt to very large parameter variations instantaneously. In fact, an SMC controller is invariant to so-called matched uncertainty. Plant uncertainties are defined as matched when they lie in the image of the plant input matrix, i.e., the uncertainties affect the plant dynamics only through the plant input channels. Thus, if the system is invariant in the presence of uncertainties such as those arising from airframe damage, there exists no need to perform failure detection, system identification, or on-line control law redesign. Indeed, it was this potential simplicity and instantaneous adaptation time that motivated the research to be described.

The most significant shortcoming with SMC designs in control applications is their inability to accommodate unmodeled parasitic dynamics. In flight control applications, these dynamics are typically those of the actuators driving the control effectors. One method which is known to help alleviate this problem is the use of asymptotic observers. ${ }^{13}$ A previous work by the authors demonstrates an observer-based SMC for a SISO flight control application and introduces a frequency domain approach for designing the sliding manifold. ${ }^{14}$ This approach has been extended to a MIMO observer-based SMC controller for an aircraft with stable 
unaugmented dynamics. ${ }^{15}$ In the current work, the unstable highly-coupled vehicle dynamics of the tailless Innovative Control Effector (ICE) fighter aircrait are addressed, and a frequency domain procedure for the important selection of observer eigenvalues is presented. In addition, a method of observer loop shaping which is equivalent to model reference hedging is introduced.

\section{MIMO Sliding Mode Control}

\section{Overview of SMC}

There are several excellent survey articles regarding SMC theory and applications. ${ }^{13,16-20}$ Hence, only a brief, simplified overview is given here, with emphasis on implementation and design issues. The key properties of a sliding mode controller are well known and are reviewed here without proof. ${ }^{21}$

a.) While on the sliding mode, the system dynamics are invariant to matched uncertainty.

b.) The hypersurface that describes $\sigma=0$ defines the transient response of the system during the sliding mode.

c.) While on the sliding mode, the trajectory dynamics are of a lower order than the original model.

Consider the uncertain system with $m$ inputs and $n$ states given by:

$$
\dot{x}(t)=\mathbf{A}(x, t)+\mathbf{B}(x, \mathrm{t}) u(t)+f(t, x, u)
$$

where $f$ represents the parameter uncertainties present in the system and is assumed to be unknown but bounded by some known function of time, system state, and control vectors. In simple terms, the objective of SMC is to define

a.) $m$ sliding surfaces or manifolds, represented in vector for as $\sigma(x)=0$, and

b.) a variable structure control given by

$$
u(x, \mathrm{t})=\rho \operatorname{sgn}(\sigma)
$$


such that the system is driven to the sliding surface $\sigma=0$ in finite time and remains upon this surface for all subsequent time. When $\sigma=0$, a sliding mode is said to have been obtained. Equation (2) is said to describe variable structure control because the control structure is dependent upon the sign of the function $\sigma$. This problem statement implies a two-step design process. First, the sliding manifold(s) must be designed. This can be accomplished by a wide variety of approaches ranging from arbitrarily selecting desired error dynamics to an LQR-like design approach utilizing the state equations in the so-called regular form. ${ }^{21}$ A method which combines a square system feedback linearization approach ${ }^{18,22-25}$ with traditional frequency domain loop shaping is used in this work. The second step in the design process is to select $\rho$ such that the reaching condition is met. This can be done analytically using a Lyapunov stability criteria or simply by use of computational simulation.

\section{SMC Implementation Issues}

The control law in Equation (2) is undefined while $\sigma=0$; therefore, the control effort required to maintain the ideal sliding mode is discontinuous with an infinite frequency switching. This control signal is unacceptable for actual mechanical systems and a continuous implementation of a SMC is almoșt always sought. One of the most common approaches to obtain a continuous control signal is the inclusion of a so-called boundary layer near the sliding surface. This can done by replacing the signum function in Equation (2) with a finite-slope line with limits at \pm 1 as shown in Equation (3).

$$
u(x, \mathrm{t})=\rho \operatorname{sat}\left(\frac{\sigma}{\varepsilon}\right)
$$

Another implementation issue with SMC controllers is the problem of unmodeled parasitic dynamics. The interaction of these parasitic dynamics with the SMC controller (even 
when a boundary layer is used) results in chatter and very often causes system instability. A simple solution to the actuator problem might be the inclusion of the actuator in the SMC design. Unfortunately, this is difficult to implement in practice because of the increased order of the sliding manifold associated with the additional dynamics. In general, the order of the manifold will increase by the same order as the modeled actuator dynamics. This means, for a second order actuator, at least two derivatives of the output signal are required. For a real system with measurement noise, these additional derivatives make this approach unattractive. There are several other approaches which have been proposed. ${ }^{13}$ One which is both effective and intuitively appealing is the use of an asymptotic observer.

\section{Observer-Based SMC}

Asymptotic observers construct a "high-frequency by pass loop," essentially hiding the unmodeled parasitic dynamics from the SMC controller. ${ }^{13}$ Selection of the observer eigenvalues is a crucial part of the observer-based SMC design. If the observer eigenvalues (and hence gains) are too large, chatter and instability results. In addition, sensor noise becomes a problem. If the observer eigenvalues (and gains) are too small, robustness to system parameter variation is lost. A method is sought to select the observer gains. Since a MIMO square system architecture effectively decouples the control variables, it can be shown that multiple observers (one for each control variable, and each with its own eigenvalues) are desired for reducing the interaction of each sliding mode control action with the parasitic dynamics. Such an architecture is shown in Fig. 1 and is directed toward the specific flight control application to be discussed in the design example. Consider the following system definitions corresponding to Fig. 1

$$
\begin{aligned}
& x \in \Re^{\mathrm{n}}, \quad x_{a} \in \mathfrak{R}^{\mathrm{n}_{\mathrm{a}}}, \quad x_{c} \in \mathfrak{R}^{\mathrm{n}_{c}}, \quad x_{r} \in \mathfrak{R}^{\mathrm{n}_{r}}, \quad \hat{x}_{o 1} \in \mathfrak{R}^{\mathrm{n}{ }^{\circ 1}}, \quad \hat{x}_{o 2} \in \mathfrak{R}^{\mathrm{n}_{02}}, \quad \hat{x}_{0, i} \in \mathfrak{M}^{\mathrm{n}_{03}} \\
& y \in \mathfrak{R}^{\mathrm{m}_{\mathrm{y}}}, \quad y_{\mathbf{a}} \in \mathfrak{R}^{\mathrm{m}}, \quad y_{\mathbf{c}} \in \mathfrak{R}^{\mathrm{m}_{\mathbf{y}}}, \quad y_{\mathbf{r}} \in \mathfrak{R}^{\mathrm{m}_{\mathbf{y}}}, \quad \hat{y}_{1} \in \mathfrak{R}^{\mathrm{m}_{\mathrm{y}}}, \quad \hat{y}_{2} \in \mathfrak{R}^{\mathrm{m}_{\mathrm{y}}}, \quad \hat{y}_{3} \in \mathfrak{R}^{\mathrm{m}_{\mathrm{y}}} \\
& z_{1} \in \mathfrak{R}^{\mathrm{m}_{21}}, \quad z_{2} \in \mathfrak{R}^{\mathrm{m}_{22}}, \quad z_{3} \in \mathfrak{R}^{\mathrm{m}_{z 3}}, \quad \hat{y} \in \mathfrak{R}^{\mathrm{m}_{\mathbf{y}}}
\end{aligned}
$$


$\underline{\text { Linear Plant }}$

$\dot{x}=\mathbf{A} x+\mathbf{B} y_{\mathrm{a}}$

$y=\mathbf{C}_{\mathbf{y}} x+\mathbf{D}_{\mathbf{y}} y_{\mathbf{a}}$

$z_{1}=\mathbf{C}_{\mathbf{z} 1} x+\mathbf{D}_{\mathbf{z} 1} y_{\mathbf{a}}$

$z_{2}=\mathbf{C}_{\mathbf{z 2}} x+\mathbf{D}_{\mathbf{z 2}} y_{\mathrm{a}}$

$z_{3}=\mathbf{C}_{\mathbf{z} 3} x+\mathbf{D}_{\mathbf{z 3}} y_{\mathrm{a}}$

Reference Model

$\dot{x}_{r}=\mathbf{A}_{\mathbf{r}} x_{r}+\mathbf{B}_{r} r_{p}$

$y_{r}=\mathbf{C}_{r} x_{r}+\mathbf{D}_{r} r_{p}$

Observer 1

$\dot{\hat{x}}_{1}=\left(\mathbf{A}_{\mathrm{o} 1}-\mathrm{G}_{1} \mathrm{C}_{\mathrm{z} 1}\right) \hat{x}_{I}+\mathbf{B}_{\mathrm{o} 1} \mathrm{~K}_{\mathrm{b}} y_{\mathrm{c}}+\mathrm{G}_{1} z_{I}$

$\dot{y}_{l}=\mathbf{C}_{\mathbf{0 1}} \hat{x}_{l}$

\section{Observer 2}

$\dot{\hat{x}}_{2}=\left(\mathbf{A}_{\mathbf{0 2}}-\mathbf{G}_{\mathbf{2}} \mathbf{C}_{\mathbf{z} 2}\right) \dot{x}_{2}+\mathbf{B}_{\mathbf{0 2}} \mathbf{K} \mathbf{b} y_{\mathbf{c}}+\mathbf{G}_{\mathbf{2}} z_{2}$

$\hat{y}_{2}=\mathbf{C}_{\mathbf{0 2}} \hat{x}_{2}$

\section{$\underline{\text { Observer } 3}$}

$\dot{\hat{x}}_{3}=\left(\mathbf{A}_{\mathbf{0 3}}-\mathbf{G}_{\mathbf{3}} \mathbf{C}_{\mathbf{z} 3}\right) \hat{x}_{3}+\mathbf{B}_{\mathbf{0} 3} \mathbf{K} \mathbf{6} y_{\mathbf{C}}+\mathbf{G}_{\mathbf{3}} z_{3}$

$\hat{y}_{3}=\mathbf{C}_{\mathbf{0 3}} \hat{x}_{3}$

Output Feedback

$\hat{y}=\hat{y}_{1}+\hat{y}_{2}+\hat{y}_{3}$

\section{Actuators}

$$
\dot{x}_{a}=\mathbf{A}_{\mathbf{a}} x_{a}+\mathbf{B}_{\mathbf{a}} \mathbf{K}_{\mathbf{b}} y_{\mathbf{c}}
$$$$
y_{a}=\mathbf{C}_{\mathbf{a}} x_{a}+\mathbf{D}_{\mathbf{a}} \mathbf{K}_{\mathbf{b}} y_{\mathrm{c}}
$$

$\mathbf{C}_{01} \in \mathfrak{R}^{\mathrm{my} \times \mathrm{n}}$ with zeros in the rows corresponding to states not output by Observer 1. $\mathbf{G}_{\mathbf{1}}=$ Observer gains

$\mathbf{C}_{\mathbf{0} 2} \in \mathfrak{R}^{\mathrm{m} y \times \mathrm{n}}$ with zeros in the rows corresponding to states not output by Observer 2. $\mathbf{G}_{\mathbf{2}}=$ Observer gains

$\mathbf{C}_{03} \in \mathfrak{R}^{\mathrm{m} y \times n}$ with zeros in the rows corresponding to states not output by Observer 3. $\mathbf{G}_{\mathbf{3}}=$ Observer gains

Figure 2 shows an equivalent system with unity feedback. It is relatively simple to derive the state space representation for the system in Fig. 2 as

$$
\begin{aligned}
& \dot{x}_{e}=\mathbf{A}_{\mathbf{e}} x_{e}+\mathbf{B}_{\mathbf{e}} y_{c} \\
& y=\left[\begin{array}{ll}
\mathbf{C}_{\mathbf{y}} & \mathbf{0}_{\mathbf{m}_{\mathbf{y}} \times\left(\mathrm{n}_{\mathbf{a}}+\mathrm{n}_{\mathbf{0} 1}+\mathrm{n}_{\mathbf{0} 2}+\mathrm{n}_{\mathbf{0}}\right)}
\end{array}\right] x_{e}+\left[\mathbf{D}_{\mathbf{y}} \mathbf{K}_{\mathbf{b}}\right] y_{\mathcal{C}} \quad x_{t^{\prime}}=\left[\begin{array}{c}
x \\
x_{a} \\
\hat{x}_{I} \\
\hat{x}_{2} \\
\hat{x}_{3}
\end{array}\right] \\
& \hat{y}=\mathbf{C}_{\mathbf{e}} x_{e}
\end{aligned}
$$

where 


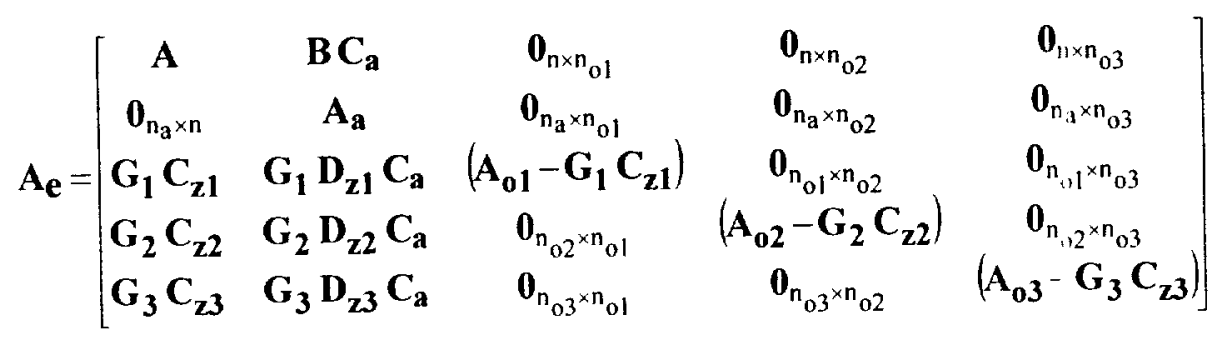

$$
\begin{aligned}
& B_{e}=\left[\begin{array}{c}
B D_{a} K_{b} \\
B_{a} K_{b} \\
B_{01} K_{b}+G_{1} D_{z 1} D_{a} K_{b} \\
B_{02} K_{b}+G_{2} D_{z 2} D_{a} K_{b} \\
B_{03} K_{b}+G_{3} D_{z 3} D_{a} K_{b}
\end{array}\right] \\
& \mathbf{C}_{\mathbf{e}}=\left[\begin{array}{lllll}
\boldsymbol{0}_{\mathrm{m}_{y} \times \mathrm{n}} & \mathbf{0}_{\mathrm{m}_{y} \times \mathrm{n}_{\mathrm{a}}} & \mathrm{C}_{\mathrm{o1}} & \mathrm{C}_{\mathrm{o} 2} & \mathrm{C}_{\mathbf{0 3}}
\end{array}\right]
\end{aligned}
$$

It is instructive to now examine the transfer functions $\frac{\hat{y}_{y}}{y_{c}}(s)$ and compare them with both the original nominal plant transfer functions with no actuator and the plant with the actuator. Figure 3 shows the Bode plots of $\frac{\hat{y}}{y_{c}}(s)$ for the ICE vehicle, where $y==\alpha=$ angle of attack. The nominal plant transfer function is shown along with the observed fecdback signal for various observer bandwidths. Several observations are worth noting. First of all, while the addition of actuator dynamics (with no observer) changes the apparent relative order for the SMC, the apparent relative order of the observed signal is the same as the nominal plant with no actuators. This is important because the sliding manifold is one degree less than the relative order of the plant and is not tolerant to changes in relative order. Second, fast observers (those with eigenvalues far into the left half plane) display a characteristic distortion in the Bode magnitude curve of $\frac{\hat{y}}{y_{c}}(s)$ near the frequency of the actuator dynamics and a large increase in phase lag. Third, by slowing down the observer, the distortion in the magnitude curve and the large phase lag can be greatly 
reduced. This fact can be used to help determine the best observer gains. Similar Bode plots are examined for each controlled variable in the square system architecture.

\section{Model Reference Hedging}

Observer-based SMC helps deal with the problem of unmodeled parasitic dynamics. However, the addition of the observer does not directly address the issue of control saturation. The observer does help with rate saturation to some degree because of phase lag reductions at medium to high frequencies. However, position saturation is still an issue-especially when the system does not have redundant control effectors. Initially, in an attempt to deal with control saturation, a method called model reference hedging was pursued. The concept of hedging has been successfully demonstrated in a dynamic inversion design approach. ${ }^{26}$ The concept in words is this: "The reference model is moved backwards (hedged) by an estimate of the amount the plant did not move due to system characteristics the control designer does not want the adaptive control element to 'know' about." 26 The actual accelerations are subtracted from the expected accelerations (assuming no actuators). This difference represents the amount of desired acceleration which was not achieved due to the actuators and should capture nonlinear saturation of the actual actuators. This difference is then subtracted from the reference model acceleration. Since all this is done in a dynamic inversion setting, these accelerations are pseudo-commands for the dynamic inversion controller, and Johnson et al. call this "Pseudo-Control Hedging." The concept has great merit and is very successful in such applications. ${ }^{26}$

In order to employ this method in an SMC design, some modification to the implementation is required. Rather than subtracting the acceleration difference from the reference model pseudo-command, the controlled variable output is subtracted from the expected output (through a nominal system with no actuators). This signal ( $\mathrm{y}_{\mathrm{h}}$ ) represents the amount of unachieved performance due to the presence of the actuator. The signal $y_{h}$ passes through a 
hedge gain $\left(\mathrm{K}_{\mathrm{h}}\right)$ and is subtracted directly from the second order model reference states as shown in Fig. 4.

In order to investigate how hedging affects the system, an analytical expression for the system with hedging is needed. Consider, initially, a model architecture as shown in Fig. 5. In this system, the hedge plant, $\mathrm{G}_{\mathrm{h}}$, is simply the nominal plant model (assuming it is stable). The subsystem block labeled "Hedged Reference Model" is the subsystem shown in Fig. 4.. Since hedging occurs on individual control variable channels, it is possible to write the transfer function for the hedged reference model. It can be shown that this transfer function (for a $2^{\text {nd }}$ order reference model) is given by

$$
\begin{aligned}
x_{r h} & =\left(\frac{m_{0}}{s^{2}+m_{1} s+m_{0}}\right) x_{c}-\left(\frac{2 s^{2}+m_{1} s}{s^{2}+m_{1} s+m_{0}}\right) K_{h} y_{h} \\
& \equiv G_{r}(s) x_{c}-G_{f}(s) K_{h} y_{h}
\end{aligned}
$$

Equation (6) indicates that the hedged reference signal consists of two parts, a reference model part and a hedge signal passing through a "hedge filter," $\mathrm{G}_{1}$. With this definition, the block diagram can be redrawn as shown in Fig. 6. With the following hedge system definitions

$$
\begin{aligned}
& \underline{\text { Hedge Plant }} \\
& \dot{x}_{h}=\mathbf{A}_{\mathbf{h}} x_{h}+\mathbf{B}_{\mathbf{h}} \mathbf{K}_{\mathbf{b}} y_{\mathbf{c}} \quad \dot{x}_{f}=\mathbf{A}_{\mathbf{f}} x_{f}+\mathbf{B}_{\mathbf{f}} \mathbf{K}_{\mathbf{h}} y_{\mathbf{h}} \\
& y_{h}=\mathbf{C}_{\mathbf{h}} x_{h}+\mathbf{D}_{\mathbf{h}} \mathbf{K}_{\mathbf{b}} y_{\mathbf{c}} \quad y_{f}=\mathbf{C}_{\mathbf{f}} x_{f}+\mathbf{D}_{\mathbf{f}} \mathbf{K}_{\mathbf{h}} y_{\mathbf{h}} \\
& \text { Output Feedback } \\
& \hat{y}_{h}=\hat{y}_{I}+\hat{y}_{2}+\hat{y}_{3}+y_{f}
\end{aligned}
$$

the equivalent unity feedback system (as in Fig. 2) is defined in state space as 
$\dot{x}_{e}=\mathbf{A}_{\mathbf{e}} x_{e}+\mathbf{B}_{\mathbf{e}} y_{\mathcal{C}}$

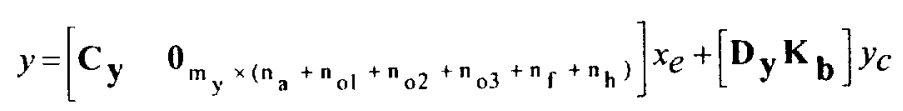

$x_{e}=\left[\begin{array}{c}x \\ x_{a} \\ \hat{x}_{1} \\ \hat{x}_{2} \\ \hat{x}_{3} \\ x_{f} \\ x_{h}\end{array}\right]$

$\hat{y}_{h}=\mathbf{C}_{\mathbf{e}} x_{e}+\mathbf{D}_{\mathbf{e}} y_{c}$

where

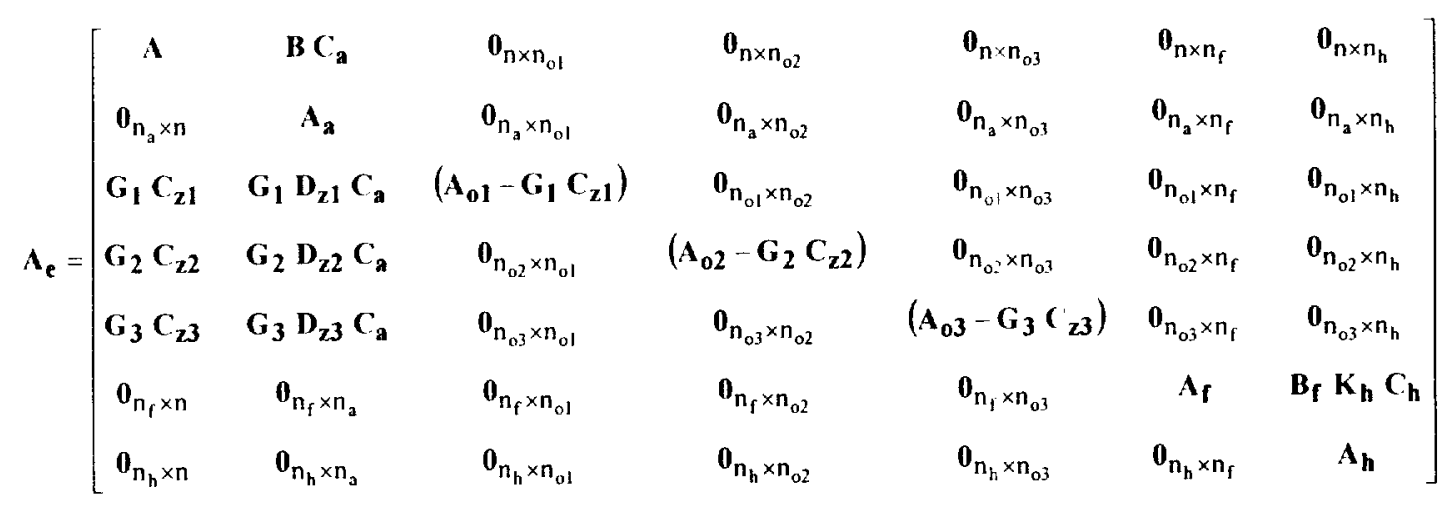

$B_{e}=\left[\begin{array}{c}B D_{a} K_{b} \\ B_{a} K_{b} \\ B_{01} K_{b}+G_{1} D_{z 1} D_{a} K_{b} \\ B_{02} K_{b}+G_{2} D_{22} D_{a} K_{b} \\ B_{03} K_{b}+G_{3} D_{z 3} D_{a} K_{b} \\ B_{f} K_{h} D_{h} K_{b} \\ B_{h} K_{b}\end{array}\right]$

Y

$C_{e}=\left[\begin{array}{lllllll}0_{m_{y} \times n} & 0_{m_{y} \times n_{a}} & C_{o 1} & C_{02} & C_{03} & C_{f} & D_{f} K_{h} C_{h}\end{array}\right]$

$D_{e}=D_{f} K_{h} D_{h} K_{b}$

Now the effects of hedging can be examined on the Bode plots of the transfer functions

$\frac{\hat{y}_{h}}{y_{c}}(s)$ as was done with the observer. After examining several different systems, it was noted 
that the hedge signal has basically the same loop shape for each system. It resembles a derivative at low frequencies; it peaks, and then rolls off at the relative order of the plant at high frequencies. This is not surprising considering the hedge filter has the same form in all cases-it resembles a high-pass filter. All real plants have a high frequency roll-off. When these two are placed in series, the result is a characteristic "hump" shape. The question then is this: can the same beneficial effects of hedging be achieved with a simplified "equivalent hedge" transfer function? This is highly desirable because, in its current form, hedging can only be used with a stable plant.

Consider a hedge filter of the form (a high-pass filter):

$$
G_{f}(s)=\frac{s}{s+a_{f}}
$$

And a hedge plant of the form (a low-pass filter):

$$
G_{h}(s)=\frac{b_{h}}{s^{2}+a_{h} s+b_{h}}
$$

The hedge plant, $G_{h}$, in Eqn. (10) has relative order 2 and is intended to be used with a system with a relative order of 1 . The extra pole is added in order to have additional high frequency roll-off. In general, the simplified model can be designed to be close to the original hedge system and will achieve the same desired effects. Note, the actuator states are not required as they are in pseudo-command hedging. ${ }^{26}$ The following technique is proposed for creating the hedge model. Begin by plotting the Bode plots of $\frac{\hat{y}_{h}}{y_{c}}(s)$ and the nominal system as before with zero hedge gain. The basic form of the desired hedge model loop shape is as follows:

(1) $+20 \mathrm{~dB} / \mathrm{dec}$ slope at low frequencies 
(2) $-20 \cdot \mathrm{r} \mathrm{dB} / \mathrm{dec}$ slope at frequencies where the actuators distort the magnitude curve $(r=$ relative degree of nominal system with no actuators $)$

(3) $\quad-20 \cdot r-20 \mathrm{~dB} / \mathrm{dec}$ slope at high frequencies

The desired hedge signal loop shape can be created (for a system with relative degree 1) using the hedge plant and hedge filter given in Eqns. (9) and (10). The pole in the hedge filter is placed at the high frequency end of the magnitude distortion and the two poles of the hedge plant are placed at the low frequency end of the distortion. A conceptual example of this is illustrated in Fig. 7. With the correct loop shape, the hedge gain is then adjusted to minimize error in the phase plot and the distortion in the magnitude plot. If the observer is very fast, or if no observer is used, there is a minimum hedge gain that will stabilize the system. Using this method of examining the Bode plots of $\frac{\hat{y}_{h}}{y_{c}}(s)$, it is easy to determine the minimum hedge gain. Examining the Bode plots will also give an indication of the upper limit for this gain. In general, if the hedge signal moves the magnitude plot of $\frac{\hat{y}_{h}}{y_{c}}(s)$ above the nominal plant magnitude plot, the closed-loop system will have unacceptable overshoot and phase lag. This can lead to instability if outer control loops are closed around the SMC system.

In MIMO applications, the cross-coupled transfer functions need to be examined as well. If cross-coupling effects are strong, the interaction of the command for one channel with the unmodeled parasitic dynamics can lead to instability in another channel. Therefore, if necessary, a hedge model for the cross-coupling term can be designed and the feedback loop properly shaped. The same guidelines introduced above are also used for the cross-coupled hedge models. This cross-term hedge signal is then added to the primary hedge signal. 
It is worth noting that the proposed hedging method no longer resembles the concept of hedging given in the initial reference work. ${ }^{26}$ Note in Fig. 11 that all the hedge signal dynamics occur in parallel with the observer loop and no longer enter the reference model. The hedging method here is really a form of observer loop shaping. In fact, this approach is similar to Loop Transfer Recovery (LTR) as used in Linear Quadratic Gaussian (LQG) control in which optimality of the observer is traded for increased stability margins. The hedge signal used here also has the effect of "tuning down" the observer at certain frequencies in order to recover desirable stability margins. It can also be shown that the hedge system is attempting to invert the actuator dynamics. This is why it is highly dependent on the actuator bandwidths. When viewed in this light, one could argue that this is simply another form of an SMC prefilter-type design. ${ }^{13}$

The preceding discussion has attempted to illustrate the effectiveness of model reference hedging and has demonstrated a design procedure--all in the frequency domain. Observers and hedging in the feedback paths allow the SMC to see the plant (nearly) without parasitic dynamics. Thus, some of the performance and robustness of the SMC design approach are recovered. Unfortunately, rigorous proofs that quantify stability and robustness for nonlinear applications are, as yet, unavailable. Some initial observations about robustness using a frequency domain approach are forthicoming. ${ }^{27}$

\section{Frequency Domain SMC Design Procedure}

The following technique is based upon the design approach offered in previous applications. ${ }^{14,15}$

(1) The vehicle model is obtained, along with an estimate of the frequency beyond which parasitic dynamics (or unstructured uncertainties) are likely to come into play. This frequency is referred to as the limit frequency in this discussion. Actuator dynamics are not included in the nominal plant model. 
(2) A reference model is chosen for each control variable channel. Since piloted flight control is of interest in the present application, this reference model should be selected with an eye towards Level I handling qualities with no pilot-induced oscillation (PIO) tendencies. This can be accomplished based upon a pilot model-based handling qualities and PIO prediction technique. $^{28,29}$

(3) The desired feedback structure of the control system is determined with a square system architecture. For example, if a roll-rate command flight control system is desired, then roll rate $\left(p_{c}\right)$ becomes the output of the reference model, and estimated roll rate $(\hat{p})$ is fed back to the SMC system from the observer. System error is then defined as $e(t)=p_{c}(t)-\hat{p}(t)$. Special care must be given to ensure the dynamics of the uncontrolled variables remain stable. This assumption is analogous to the minimum phase assumption used in feedback linearization. $^{23}$ Also, if there are redundant control effectors for the desired moments/pseudo-commands, a control distribution matrix must be defined.

(4) The sliding manifold, $\sigma$, is chosen based upon the following principles:

a) $\sigma$ is derived from a tracking error expression as

$$
\sigma=e(t) \eta^{-1}+K_{p-2} e(t)^{p-2}+K_{0} e(t)+K_{-1} \int e(t) d t
$$

where $p$ is the relative order of the system, i.e., the number of times the vehicle output must be differentiated for the input to appear. Note that the $(\mathrm{p}-1)^{\mathrm{st}}$ derivative of the error signal is included in the definition of $\sigma$. An integral term also appears in Eqn. (11) to counter the steady-state bias often created with the use of a boundary layer. 
b) Recognizing that a boundary layer is to be implemented, the control law is expressed as a linear transfer function, assuming the boundary layer is large enough to remain within the linear region of the saturation element.

$$
\begin{aligned}
\mathrm{u}(\mathrm{s}) & =\frac{\rho}{\varepsilon} \sigma \\
& \left.=\mathrm{K}_{\rho}\left(\mathrm{s}^{\mathrm{p}-1}+\mathrm{K}_{\mathrm{p}-2} \mathrm{~s}^{\mathrm{p}-2}+\mathrm{K}_{0}+\frac{\mathrm{K}}{\mathrm{s}}\right) \mathrm{L}\right) \mathrm{e}(\mathrm{s})
\end{aligned}
$$

The parameters $K_{i}$ are chosen to provide desirable properties in the frequency domain. This means creating a loop transmission with broad $\mathrm{K} / \mathrm{s}$-like characteristics around crossover. ${ }^{30}$ This will always be possible since enough derivatives are included in Eqn. (12) to create exact $\mathrm{K} / \mathrm{s}$ characteristics beyond a certain frequency (at least as high as the limit frequency). Parasitic dynamics are deliberately excluded in this formulation. This step will involve obtaining an estimate of $\mathrm{K}_{\rho}$, as this value will determine the crossover frequency of the loop transmission. This crossover frequency is selected to provide acceptable stability margins as obtained from a Bode plot of the loop transmission, but using a value of $\mathrm{K}_{\mathrm{p}}$ at least as large as the largest amplitude limit of any of the control effectors. The latter criterion is included to accommodate maximum trim positions of the control effectors. As opposed to typical designs involving loop shaping, very high crossover frequencies may result from this step. Indeed these frequencies may be well beyond the limit frequency. This result is of no immediate concern. If a MIMO system is being designed, a classic sequential loop closure technique is used, thereby sequentially and independently determining the coefficients for each sliding manifold. 
(5) Using the $K_{i}$ 's just determined in the definition of the sliding function, the existence of a sliding mode is verified in the inner loops using a true SMC. This step is completed without the observer, actuators, reference model or pilot model, i.e., assuming that no outer-loop is being utilized. If necessary, $\rho$ is increased until sliding behavior is created. The initial value of $\rho=K_{\rho}$ obtained in step (4b) should be considered a lower limit in this process. While an analytical approach to determine $\rho$ is certainly possible here, a more expedient route of establishing the sliding mode using a computer simulation of the system is also possible. Near perfect tracking in the face of large parameter variations should be observed. The control signal, however, will exhibit very high frequency switching.

(6) A boundary layer is included in the controller by replacing the signum function $\operatorname{sign}(\sigma)$ with the saturation function $\operatorname{sat}\left(\frac{\sigma}{\varepsilon}\right)$. While maintaining an approximate constant $\frac{\rho}{\varepsilon}=\mathrm{K}_{\rho}$, increase the boundary layer thickness, $\varepsilon$, until no high frequency switching is evident. Again, a simulation of the SMC system is a convenient way of finding this $\varepsilon$. Near-perfect tracking (with a continuous control signal) in the face of large parameter variations should be observed.

(7) Parasitic dynamics are included in the model. The SMC controller will very likely be unstable at this juncture.

(8) An asymptotic observer is created for each control variable channel as discussed above.

(9) A hedge model is designed as described above. 
(10) The frequency domain characteristics of the open and closed-loop SMC system with observer, boundary layer and reference model are examined to ensure that stability of the linear system is in evidence.

A criticism that can be levied against SMC design procedures such as the one discussed here is that, after introduction of a non-adaptive boundary layer, onc has essentially created little more than a MIMO PID controller. However, an examination of the characteristics of the equivalent unity feedback systems proves this assumption incorrect. Consider again the SMC system of Fig. 1. Focusing upon any one of the closed-loop transfer functions of the MIMO system, $\frac{\mathrm{y}}{\mathrm{r}}(\mathrm{s})$, one can create an equivalent unity feedback loop transmission as

$$
L=\frac{(y / r)}{1-(y / r)}
$$

This can then be used to create an equivalent serial compensation element, $G_{c_{e}}(s)$, which represents the combination of the controller and the observer.

$$
G_{c_{\mathrm{e}}}=\frac{L}{\left(y / y_{c}\right)}
$$

By maintaining the SMC and observer parameters constant, but introducing changes in the vehicle or actuator dynamics, it can be shown that $G_{c_{e}}(s)$ will vary in a manner that attempts to compensate for changes in the vehicle dynamics. This behavior is attributable to the presence of the observer(s) in the feedback loops. Of course, the changes in $G_{c_{s}}(s)$ are instantaneous.

\section{Design Example: ICE MIMO Linear 6-DOF Model}

\section{ICE Vehicle}

The Integrated Control Effectors (ICE) aircraft model has been developed by Lockheed under an Air Force Research Laboratories (AFRL) sponsored program ${ }^{31}$ and is the vehicle of 
choice for many controls applications in the current literature. ${ }^{1,2,11,23,32-38}$ It is a single engine, multi-role, supersonic, tailless fighter aircraft with a 65 degree sweep delta wing (see Fig. 8). As illustrated in Fig. 9, the conventional control effectors include elevons, symmetric pitch flaps, and outboard leading edge flaps. The innovative control effectors include pitch and yaw thrust vectoring, all moving tips, and spoiler slot deflectors. The all moving tips and spoiler slot deflectors have zero lower deflection limits.

The static aerodynamic force and moment data were collected by NASA Langley Research Center and AFRL using wind tunnel tests with a $1 / 18^{\text {th }}$ scale model. Additional wind tunnel tests during Phase II of the ICE program provide updated data for simulation models.' There are strong multi-axis effects and highly nonlinear interactions between the close-coupled control surfaces. The full nonlinear simulation of the ICE vehicle is proprietary; however, linearized models at various flight conditions (which do not include nonlinear effector interactions) have been made available to this research directly from the NASA Langley Research Center.

\section{SMC Design}

The SMC design considers simultaneous control of longitudinal and lateral degrees of freedom. The response variables ane angle of attack, $\alpha$, roll-rate about the velocity vector, $\mathrm{p}_{s}$, and sideslip, $\beta$. Decoupled tracking performance is desired.

(1) The flight condition used for this study is: $1 \mathrm{~g}$, wings-level, Mach No. $=0.3$, Alt $=15,000 \mathrm{ft}$. The linearized model for this flight condition contains eight states, eleven outputs, and eleven control inputs. The open-loop plant is unstable and highly coupled.

(2) Reference models for each response variable are created as well-damped secondorder systems with dynamics predicted to yield Levell handling qualities with no PIO 
tendencies. The methodology utilizing a pilot structural model is employed. ${ }^{28}$ The reference models so obtained are:

$$
\begin{aligned}
& \mathrm{G}_{\mathrm{a}}(\mathrm{s})=\frac{\alpha_{\mathrm{r}}}{\alpha_{\mathrm{c}}}(\mathrm{s})=\frac{100}{\left(\mathrm{~s}^{2}+20 \mathrm{~s}+100\right)} \\
& \mathrm{G}_{\mathrm{p}}(\mathrm{s})=\frac{\mathrm{p}_{\mathrm{r}}}{\mathrm{p}_{\mathrm{c}}}(\mathrm{s})=\frac{100}{\left(\mathrm{~s}^{2}+20 \mathrm{~s}+100\right)} \\
& \mathrm{G}_{\beta}(\mathrm{s})=\frac{\beta_{\mathrm{r}}}{\beta_{\mathrm{c}}}(\mathrm{s})=\frac{100}{\left(\mathrm{~s}^{2}+25 \mathrm{~s}+100\right)}
\end{aligned}
$$

(3) A square system architecture is defined as shown in Fig. 10. As shown, the feedback structure of the flight control system consists of angle of attack command and hold, roll-rate command, and sideslip command and hold. Analytical models of the human pilot controlling the angle of attack $(\alpha)$ and roll-attitude $(\phi)$ loops are developed and included in the Simulink simulation of the pilot/vehicle system. ${ }^{28}$ A common crossover frequency of $1.5 \mathrm{rad} / \mathrm{s}$ are chosen for the $\alpha$ and $\phi$-loops. The $\beta$ loop is not closed by the pilot, i.e., it is assumed that the pilot flies the vehicle with "feet on the floor." The controller to be designed generates pseudo-commands for angle of attack, stability axis roll rate, and sideslip angle. These demands are allocated to the eleven control effectors using a pseudo-inverse approach. ${ }^{39}$ If the preferred actuator positions are assumed to be zero, the solution to the control allocation problem using a pseudo-inverse is

$$
\mathbf{B}_{\mathrm{k}}=\mathbf{W}_{u}^{-1} \mathbf{B}^{T}\left(\mathbf{B} \mathbf{W}_{u}^{-1} \mathbf{B}^{T}\right)^{-1}
$$

where the weighting matrix, $\mathbf{W}_{\mathrm{u}}$, is assumed to be identity. The resulting control distribution matrix, $B_{k}$, is an $11 \times 3$ matrix with constant elements. Noncorrelated sum-of-sines inputs serve as tracking commands to the pilot in the $\phi$ and $\alpha$ loops. $\beta$ command is zero.

(4) The system (without actuators) has a relative order of 1 for all three control variables. Therefore, the form of the sliding manifolds are 


$$
\sigma=K_{0} e+K_{-1} \int e \mathrm{~d} \tau ; \quad e=\left\{\left(\alpha_{r}-\hat{\alpha}\right),\left(p_{\mathrm{r}}-\hat{\mathrm{p}}\right),\left(\beta_{\mathrm{r}}-\hat{\beta}\right)\right\}^{\mathrm{T}}
$$

The control laws, assuming the use of a boundary layer, thell can be expressed in linear form, resulting in one zero to be placed and a gain to set for each loop during the loop-shaping design. Using a traditional sequential loop shaping technique, the parameters of Eqn. (17) are determined--with roll rate being the first loop to be closed, followed by $\alpha$; then $\beta$. The designed manifolds are given in Eqn. (18), and the design Bode plots for the last of the loop closures ( $\beta$-loop) are shown in Fig. 11. A crossover frequency of $\omega=1000 \mathrm{rad} / \mathrm{s}$ is set for each channel.

$$
\begin{aligned}
& u_{c \alpha}(s)=5000\left(\frac{s+10}{s}\right) \\
& u_{c p}(s)=1000\left(\frac{s+20}{s}\right) \\
& u_{c \beta}(s)=5000\left(\frac{s+10}{s}\right)
\end{aligned}
$$

(5) Sliding behavior is verified by simulation. As expected, the SMC provides nearperfect tracking and is invariant to the system parameter changes. The control output shows the classic high frequency switching. The controller achieves decoupled tracking of $\alpha, p_{s}$ and $\beta$, as desired.

(6) Next, the boundary layer is increased until a continuous control signal is achieved. For this model, $\varepsilon=1$ is chosen for all three channels. Again, the performance is excellent, even in the face of large system failures.

(7) The next step is the inclusion of the actuators. The actuators are second order with rate and position limits. As expected, the nominal system immediately goes unstable.

(8)-(9) The next steps are to design the observers and hedge models. In this procedure, the eigenvalues of the observers are chosen to be real, and nearly identical. Using the frequency 
domain approach introduced earlier, it is seen that (without hedging) the $\alpha$-channel observer must have eigenvalues of $10 \mathrm{rad} / \mathrm{s}$ or less; the p-channel observer can have eigenvalues as large as $100 \mathrm{rad} / \mathrm{s}$; and the $\beta$-channel must have eigenvalues of $5 \mathrm{rad} / \mathrm{s}$ or less. It appears that hedging will help in the $\alpha$-channel, is unnecessary in the p-channel. and is probably required in the $\beta$-channel. Three cross-coupling hedge models were found to be necessary in the design. The parameters chosen for the observer and hedging models are shown in Table 1. The design Bode plots for each primary channel are given in Figs. 12 - 14.

\section{Simulation Results}

To illustrate the robustness of the observer-based SMC design, the system is exercised with measurement noise, system failure, and the pilot model in the loop. The Simulink simulation is run with an ODE2 solver using a fixed time step of $\Delta t=0.0005 \mathrm{sec}$. Vehicle failure occurs at $t=20 \mathrm{sec}$. Vehicle failure is defined as:

(1) Plant failure (A-matrix is multiplied by 2 -with the exception of the elements describing kinematic relationships; B-matrix is multiplied by 0.75 )

(2) All actuators experience a $50 \mathrm{~ms}$ time delay

(3) Left elevon rate limits are reduced from $150 \mathrm{deg} / \mathrm{s}$ to $10 \mathrm{deg} / \mathrm{s}$; position limits are reduced from $\pm 30 \mathrm{deg}$ to $\pm 15 \mathrm{deg}$

(4) Symmetric pitch flap undamped natural frequency is reduced from $63 \mathrm{rad} / \mathrm{s}$ to $10 \mathrm{rad} / \mathrm{s}$

(5) Left leading edge flap is jammed at $+5 \mathrm{deg}$

(6) Pitch nozzle actuator undamped natural frequency is reduced from $39 \mathrm{rad} / \mathrm{s}$ to $10 \mathrm{rad} / \mathrm{s}$ 
(7) Yaw nozzle actuator undamped natural frequency is reduced from $39 \mathrm{rad} / \mathrm{s}$ to $10 \mathrm{rad} / \mathrm{s}$

The resulting outer loop tracking is shown in Fig. 15. After system failure, tracking is noticeably degraded, but the vehicle remains stable. The actuator deflections are not shown, however, it is seen that nearly all the actuators are in nearly constant rate saturation after the failure. Predictions of handling qualities and PIO susceptibility for the SMC system after the failure can be made using the pilot/vehicle analysis technique. ${ }^{28,40}$ As seen in Figs. 16 and 17, Level I handling qualities are still predicted for the $\alpha$-tracking task, although PIO susceptibility is increased. Level II handling qualities are predicted for the $\phi$-tracking task, and PIO tendencies are moderately strong. In viewing these results, certain observations are in order. First of all, this is a significant system failure, and it is remarkable that the controller is able to maintain stability. Second, the input maneuvers are very aggressive. After a failure of this magnitude, a pilot would not be commanding such a demanding profile. Third, the HQ and PIO level predictions assume a static pilot model. In actuality, a real pilot would compensate for the perceived change in vehicle dynamics. Therefore, the results shown above are conservative. One can compare these results with a classic loop-shaped controller. As Fig. 18 indicates, this design goes unstable shortly after the failure occurs.

\section{Conclusions}

Using asymptotic observers with SMC has been shown to help mitigate the adverse effects of parasitic dynamics, and a design method for choosing observer gains has been presented. A form of model reference hedging has been shown to be equivalent to an SMC prefilter which helps shape the feedback loop--thus partially removing the effects of actuator dynamics. A complete design procedure has been presented which incorporates a frequency domain approach to select 1) the sliding manifold, 2) the observer eigenvalues, and 3) the 
hedging dynamics. The approach was successfully applied to the design of a flight control system for a model of the Innovative Control Effector fighter aircraft. Stability and performance robustness was demonstrated in the presence of significant actuator degradation and variation in vehicle dynamics.

\section{Acknowledgements}

This research was supported by grant NCC1-01010 from NASA Langley Research Center. Dr. Barton Bacon serves as the grant technical manger. Support is also provided by the USAF in sponsoring Major Wells' graduate studies.

\section{References}

'Eberhardt, R. L., and Ward, D. G., "Indirect Adaptive Flight Control of a Tailless Fighter Aircraft," Proceedings of Guidance, Navigation, and Control Conference, AIAA-994042, AIAA, Portland, OR, 9-11 Aug 1999.

${ }^{2}$ Eberhardt, R. L., and Ward, D. G., "Indirect Adaptive Flight Control Interactions," International Journal of Robust and Nonlinear Control, Vol. 9, 1999, pp. 1013-1031.

${ }^{3}$ Pachter, M., Chandler, P. R., and Mears, M., "Reconfigurable Tracking Control with Saturation," Journal of Guidance, Control, and Dynamics, Vol. 18, No. 5, 1995, pp. 1016-1022.

${ }^{4}$ Ward, D. G., and Barron, R. " "A Self-Designing Receding Horizon Optimal Flight Controller," Proceedings of American Control Conference, Vol. 5, IEEE, Seattle, WA, 21-23 Jun 1995, pp. 3490-3494.

${ }^{5}$ Ward, D. G., Monaco, J. F., and Schierman, J. D., "Reconfigurable Control for VTOL UAV Shipboard Landing," Proceedings of Guidance, Navigation, and Control Conference, AIAA-99-4045, AIAA, Portland, OR, 9-11 Aug 1999. 
${ }^{6}$ Maybeck, P. S., "Multiple Model Adaptive Algorithms for Detecting and Compensating Sensor and Actuator/Surface Failures in Aircraft Flight Control Systems," International Journal of Robust and Nonlinear Control, Vol. 9, 1999, pp. 1051-1070

${ }^{7}$ Maybeck, P. S., and Stevens, R. D., "Reconfigurable Flight Control via Multiple Model Adaptive Control Methods," IEEE Transactions on Aerospace and Electronic Systems, Vol. 27, No. 3,1991 , pp. $470-480$.

${ }^{8}$ Brinker, J. S., and Wise, K. A., "Nonlinear Simulation Analysis of a Tailless Advanced Fighter Aircraft Reconfigurable Flight Control Law," Proceedings of Guidance, Navigation, and Control Conference, AlAA-99-4040, AIAA, Portland, OR, 9-11 Aug 1999.

${ }^{9}$ Brinker, J. S., and Wise, K. A., "Flight Testing of a Reconfigurable Flight Control Law on the X-36 Tailless Fighter Aircraft," Proceedings of Guidance, Navigation, and Control Conference, AIAA-2000-3941, AIAA, Denver, CO, 14-17 Aug 2000.

${ }^{10}$ Wise, K. A., Brinker, J. S., Calise, A. J., Enns, D. F., Elgersma, M. R., and Voulgaris, P. G., "Direct Adaptive Reconfigurable Flight Control for a Tailless Advanced Fighter Aircraft," International Journal of Robust and Nonlinear Control, Vol. 9, 1999, pp. 999-1012.

${ }^{11}$ Schumacher, C., "Adaptive Flight Control Using Dynamic Inversion and Neural Networks," Proceedings of Guidanide, Navigation, and Control Conference, AIAA-99-4086, AIAA, Portland, OR, 9-11 Aug 1999.

${ }^{12}$ Calise, A. J., Lee, S., and Sharma, M., "Development of a Reconfigurable Flight Control Law for the X-36 Tailless Fighter Aircraft," Proceedings of Guidance, Navigation, and Control Conference, AIAA-2000-3940, AIAA, Denver, CO, 14-17 Aug 2000. 
${ }^{13}$ Young, K. D., Utkin, V. I., and Özgüner, Ü., "A Control Engineer's Guide to Sliding Mode Control," IEEE Transactions on Control Systems Technology, Vol. 7, No. 3, 1999, pp. $328-342$.

${ }^{14}$ Hess, R. A., and Wells, S. R., "Sliding Mode Control Applied to Reconfigurable Flight Control Design," Proceedings of 40th AlAA Aerospace Sciences Meeting and Exhibit, AIAA2002-7751, AIAA, Reno, NV, 14-17 Jan 2002.

${ }^{15}$ Hess, R. A., Wells, S. R., and Vetter, T. K., "MIMO Sliding Mode Control as an Alternative to Reconfigurable Flight Control Designs," Proces'dings of American Control Conference, IEEE, Anchorage, AK, 8-10 May 2002.

${ }^{16}$ DeCarlo, R., A., Zak, S. H., and Drakunov, S. V., "Section 57.5: Variable Structure, Sliding Mode Design," The Control Handbook, Levine, W. S. eds., CRC Press, Inc., Boca Raton, FL, 1996, pp. 941-951.

${ }^{17}$ DeCarlo, R., A., Zak, S. H., and Mathews, G. P., "Variable Structure Control of Nonlinear Multivariable Systems: A Tutorial," Proceedings of the IEEE, Vol. 76, No. 3, 1988, pp. 212-232.

${ }^{18}$ Fernández, B. R., and Hedrick, J. K., "Control of Multivariable Non-Linear Systems by the Sliding Mode Method," International Journal of Control, Vol. 46, No. 3, 1987, pp. 10191040.

${ }^{19}$ Hung, J. Y., Weibing, G., and Hung, J. C., "Variable Structure Control: A Survey," IEEE Transactions on Industrial Electronics, Vol. 40, No. 1, 1993, pp. 2-23.

${ }^{20}$ Utkin, V. I., "Sliding Mode Control Design Principles and Applications to Electric Drives," IEEE Transactions on Industrial Electronics, Vol. 40, No. 1, 1993, pp. 23-36. 
${ }^{21}$ Edwards, C., and Spurgeon, S. K., Sliding Mode Control, Taylor \& Francis Ltd, Bristol, PA, 1998.

${ }^{22}$ Shtessel, Y. B., Buffington, J. M., and Banda, S. S., "Multiple Timescale Flight Control Using Reconfigurable Sliding Modes," Journal of Guidance, Control, and Dynamics, Vol. 22, No. 6, 1999, pp. 873-883.

${ }^{23}$ Shtessel, Y. B., Buffington, J. M., and Banda, S. S., "Tailless Aircraft Flight Control Using Multiple Time Scale Re-Configurable Sliding Modes," Proceedings of Guidance, Navigation, and Control Conference, AIAA-99-4136, AIAA, Portland, OR, 9-11 Aug 1999, pp. 966-976.

${ }^{24}$ Shtessel, Y. B., Buffington, J. M., Pachter, M., Chandler, P. R., and Banda, S. S., "Reconfigurable Flight Control on Sliding Modes Addressing Actuator Deflection and Deflection Rate Saturation," Proceedings of Guidance, Navigation, and Control Conference, AIAA-98-4112, AIAA, Boston, MA, Aug 1998, pp. 127-137.

${ }^{25}$ Slotine, J.-J. E., and Li, W., Applied Nonlinear Control, Prentice Hall, Englewood Cliffs, NJ, 1991.

${ }^{26}$ Johnson, E. N., Calise, A. J., El-Shirbiny, H. A., and Rysdyk, R. T., "Feedback Linearization with Neural Network Augmentation Applied to X-33 Attitude Control," Proceedings of Guidance, Navigation, and Control Conference, AlAA-2000-4157, AIAA, Denver, CO, 14-17 Aug 2000, pp. 1-11.

${ }^{27}$ Wells, S. R., and Hess, R. A., "A Frequency-Domain Interpretation of Robustness in Sliding Mode Control Design with Boundary Layers," IEEE Transactions on Control Systems Technology, submitted. 
${ }^{28}$ Hess, R. A., "Unified Theory for Aircraft Handling Qualities and Adverse Aircraft-Pilot Coupling," Journal of Guidance, Control, and Dynamics, Vol. 20, No. 6, 1997, pp. 1141-1148.

${ }^{29}$ Siwakosit, W., Snell, S. A., and Hess, R. A., "Robust Flight Control Design with Handling Qualities Constraints Using Scheduled Linear Dynamic Inversion and Loop-Shaping," IEEE Transactions on Control Systems Technology, Vol. 8, No. 3, 2000, pp. 483-494.

${ }^{30}$ Maciejowski, J. M., "Chapt. 1," Multivariable Feedbuck Design, Addison-Wesley, Wokinhman, UK, 1989,

31"F/A-18A Flight Control System Design Report," McDonnell Aircraft Corp, MDC A7813, March 15, 1983.

32"Innovative Control Effectors," USAF, WL-TR-96-3043, Jan, 1996.

${ }^{33}$ Bacon, B. J., and Ostroff, A. J., "Reconfigurable Flight Control Using Nonlinear Dynamic Inversion with a Special Accelerometer Implementation," Proceedings of Guidance, Navigation, and Control Conference, AIAA-2000-4565, AlAA, Denver, CO, 14-17 Aug 2000.

${ }^{34}$ Barker, J. M., and Balas, G. J., "Flight Control of a Tailless Aircraft Via Linear Parameter-Varying Techniques," Proceedings of Guidance, Navigation, and Control Conference, AIAA-99-4133, AIAA, Portland, OR, 9-11 Aug 1999.

${ }^{35}$ Buffington, J. M., "Tailless?Aircraft Control Allocation," Proceedings of Guidance, Navigation, and Control Conference, AIAA-97-3605, AIAA, New Orleans, LA, Aug 1997, pp. 737-747.

${ }^{36}$ Buffington, J. M., Chandler, P. R., and Pachter, M., "Integration of On-Line System Identification and Optimization-Based Control Allocation," Proceedings of Guidance, Navigation, and Control Conference, AIAA-98-4487, AIAA, Boston, MA, Aug 1998, pp. 17461756. 
${ }^{37}$ Buffington, J. M., and Shtessel, Y. B., "Saturation Protection for Feedback Linearizable Systems using Sliding Mode Theory," Proceedings of Americun Control Conference, Vol. 2, IEEE, Philadelphia, PA, 24-26 Jun 1998, pp. 1028-1032.

${ }^{38}$ Buffington, J. M., Chandler, P. R., and Pachter, M., "On-Line System Identification for Aircraft with Distributed Control Effectors," International .Journal of Robust and Nonlinear Control, Vol. 9, 1999, pp. 1033-1049.

${ }^{39}$ Page, A. B., and Steinberg, M. L., "A Closed-Loop Comparison on Control Allocation Methods," Proceedings of Guidance, Navigation, and Control Conference, AIAA-2000-4538, AIAA, Denver, CO, 14-17 Aug 2000.

${ }^{40}$ Hess, R. A., Zeyada, Y., and Heffley, R. K., "Modeling and Simulation for Helicopter Task Analysis," Proceedings of American Helicopter Society 57th Annual Forum, Washington, D.C, 9-11 May 2002. 


\section{Figure Captions}

Figure 1 Multiple observer-based SMC system

Figure 2 Unity feedback equivalent of Fig. 1

Figure 3 Bode plots, $\frac{\hat{\alpha}}{\mathrm{u}_{\mathrm{c} \alpha}}$, ICE, various observer eigenvalues

Figure $42^{\text {nd }}$ order hedged reference model

Figure 5 Hedged system architecture

Figure 6 Equivalent hedged system architecture

Figure 7 Creating the hedge model, $\frac{\bar{y}_{h}}{y_{c}}$

Figure 8 Innovative Control Effector (ICE) vehicle

Figure 9 ICE control effectors

Figure 10 Simulink diagram of the ICE pilot/vehicle/SMC system

Figure 11 Loop transmission for $\beta$-loop of step (4) in ICE SMC design, all other loops closed

Figure 12 Bode plots, $\frac{\bar{\alpha}_{h}}{u_{c a}}$, ICE hedge design

Figure 13 Bode plots, $\frac{\hat{\mathrm{p}}_{\mathrm{h}}}{\mathrm{u}_{\mathrm{cp}}}$, ICE hedge design

Figure 14 Bode plots, $\frac{\widehat{\beta}_{h}}{u_{c \beta}}$, ICE hedge design

Figure 15 Outer loop tracking ( $\alpha, \phi$, and $\beta$ ), ICE, with SMC, actuators, observers, hedging and noise, failure at $\mathrm{t}=20 \mathrm{sec}$

Figure $16 \alpha$-tracking task HQ and PIO predictions, ICE failed system

Figure $17 \phi$-tracking task HQ and PIO predictions, ICE failed system

Figure 18 Outer loop tracking $(\alpha, \phi$, and $\beta$ ), ICE, with loop-shaped controller, actuators and noise, failure at $\mathrm{t}=20 \mathrm{sec}$ 
Table 1: Observer and Hedge Models for ICE

\begin{tabular}{|c|c|}
\hline Channel & Observer Poles \\
\hline$\alpha$ & $\lambda=-10.0,-10.1,-10.2,-10.3,-10.4,-10.5,-10.6,-10.7$ \\
\hline$p_{s}$ & $\lambda=-40.0,-40.1,-40.2,-40.3,-40.4,-40.5,-40.6,-40.7$ \\
\hline \multirow[t]{2}{*}{$\beta$} & $\lambda=-1.0,-1.1,-1.2,-1.3,-1.4,-1.5,-1.6,-1.7$ \\
\hline & Hedge Model \\
\hline$\alpha_{\mathrm{h}}$ & \multirow{2}{*}{$\left.\frac{s}{s+10}\right)\left(\frac{4}{s^{2}+4 s+4}\right)$} \\
\hline $\mathrm{u}_{\mathrm{c} \alpha}$ & \\
\hline $\mathrm{p}_{\mathrm{h}}$ & \multirow{2}{*}{$\left(\frac{s}{s+50}\right)\left(\frac{4}{s^{2}+4 s+4}\right)$} \\
\hline$\overline{u_{\mathrm{cp}}}$ & \\
\hline$\beta_{\mathrm{h}}$ & \multirow{2}{*}{$\left(\frac{s}{s+5}\right)\left(\frac{2}{s^{2}+3 s}+2\right)$} \\
\hline$\overline{\mathrm{u}_{\mathrm{c \beta}}}$ & \\
\hline$\alpha_{\mathrm{h}}$ & \multirow{2}{*}{$0.0001\left(\frac{s}{s+10}\right)\left(\frac{4}{s^{2}+4 s+4}\right) \alpha$ cross term } \\
\hline$\overline{\mathrm{u}_{\mathrm{c} \beta}}$ & \\
\hline$\underline{p_{\mathrm{h}}}$ & \multirow{2}{*}{$-0.2\left(\frac{s}{s+50}\right)\left(\frac{64}{s^{2}+16 s+64}\right) p_{s}$ cross term } \\
\hline $\mathrm{u}_{\mathrm{c} \beta}$ & \\
\hline \multirow{3}{*}{$\frac{\beta_{h}}{u_{c p}}$} & \multirow{2}{*}{$0.02\left(\frac{s}{s+5}\right)\left(\frac{1}{s^{2}+2 s+1}\right) \beta$ cross term } \\
\hline & \\
\hline & Hedge Gain \\
\hline$\alpha$ & 1.0 \\
\hline$p_{\mathrm{s}}$ & 2.0 \\
\hline$\beta$ & 10.0 \\
\hline
\end{tabular}




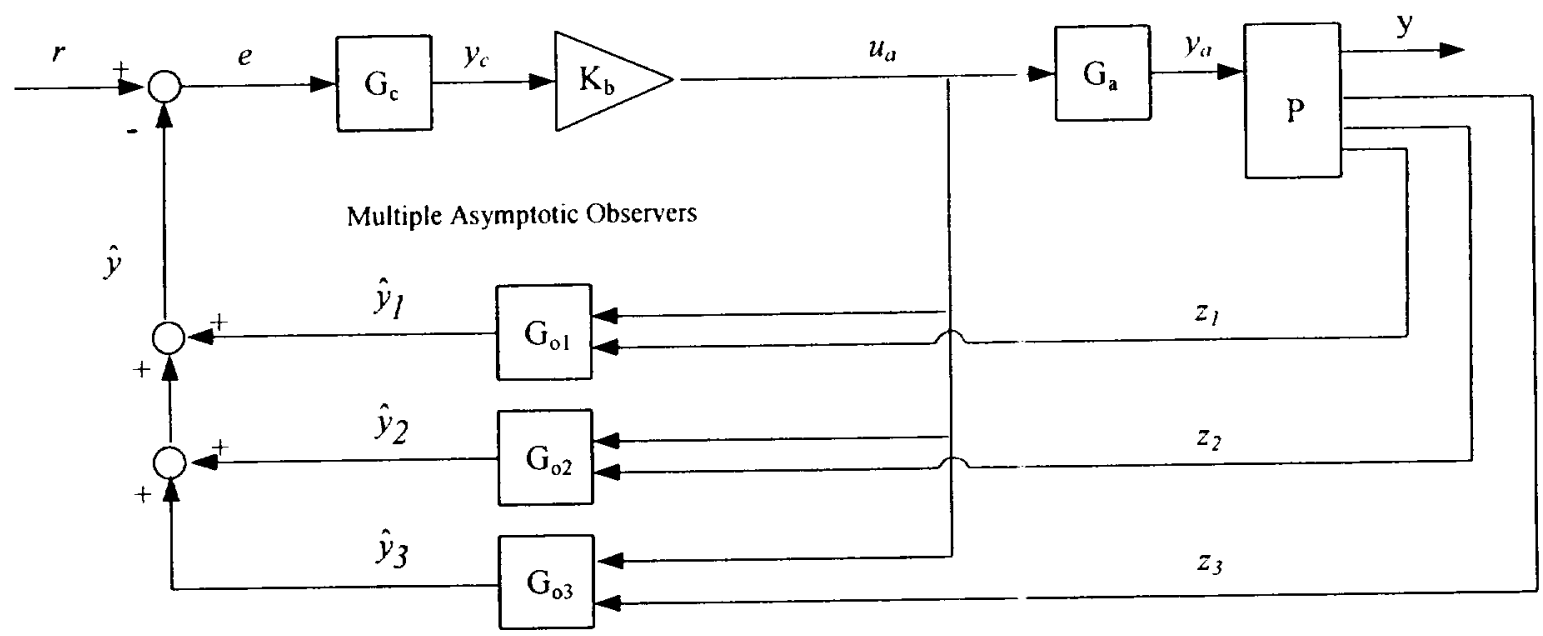

Figure 1: Multiple observer-based SMC system 


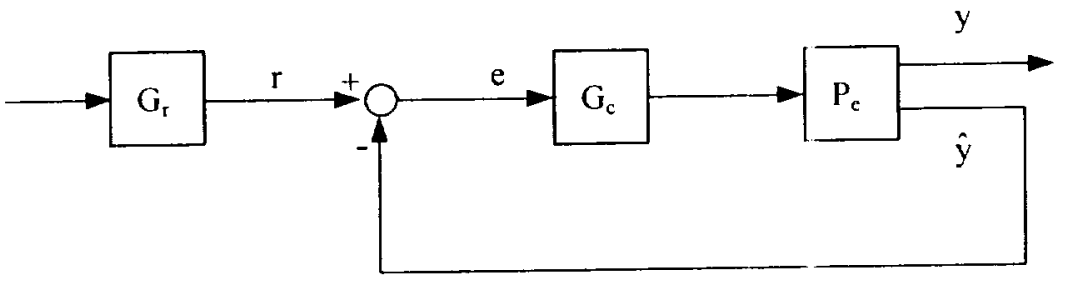

Figure 2: Unity feedback equivalent of Fig. 1

Y 

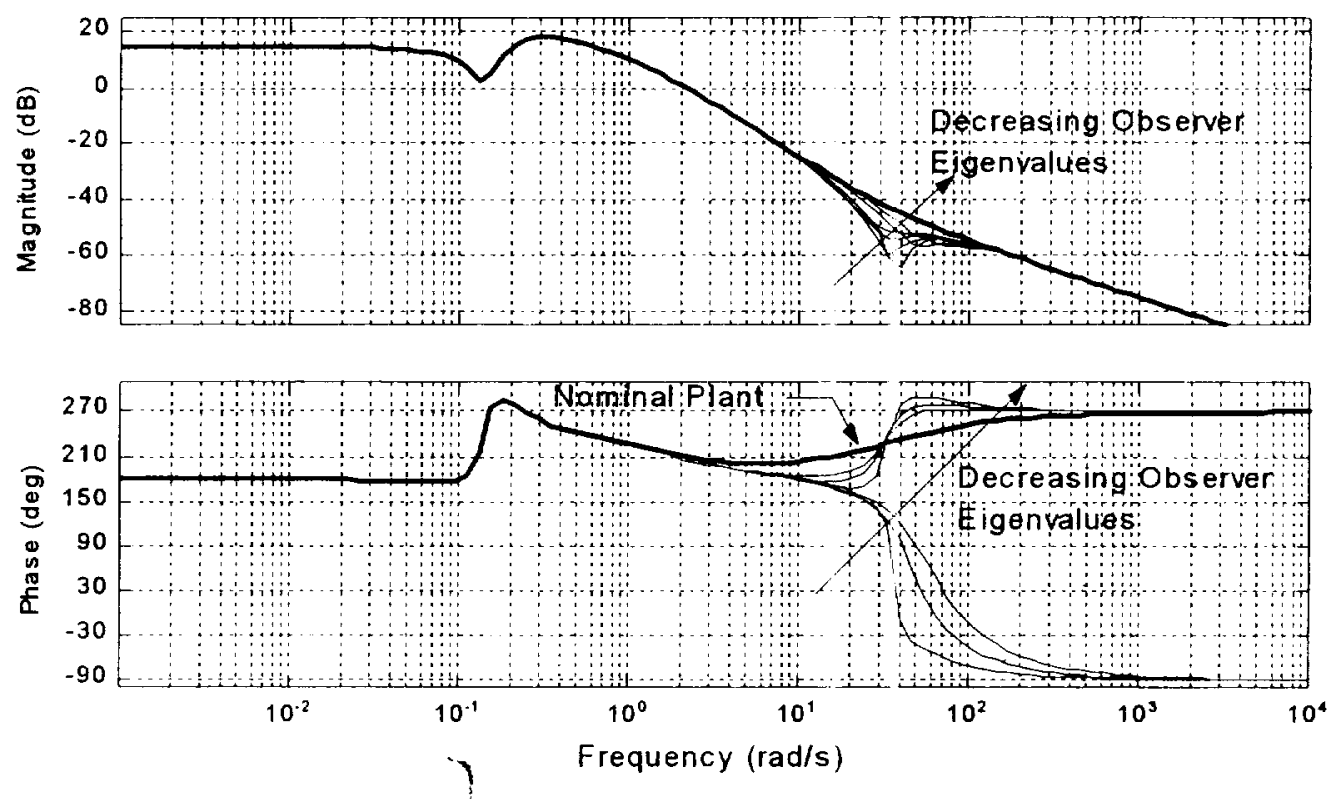

Figure 3: Bode plots, $\frac{\hat{\alpha}}{\mathrm{u}_{\mathrm{c} \alpha}}$, ICE, various observer eigenvalues 


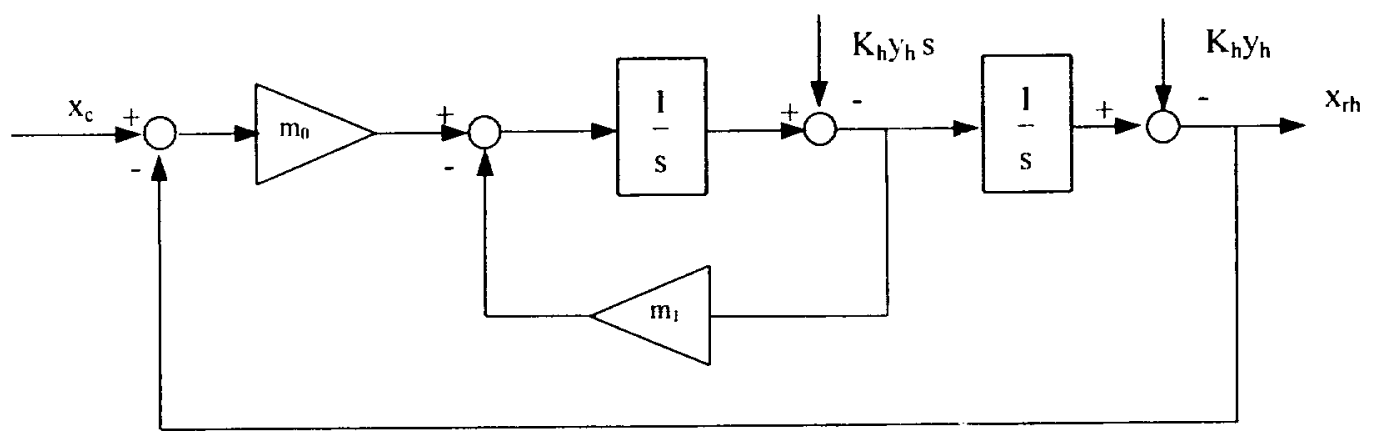

Figure 4: $2^{\text {nd }}$ Order hedged reference model

Y 


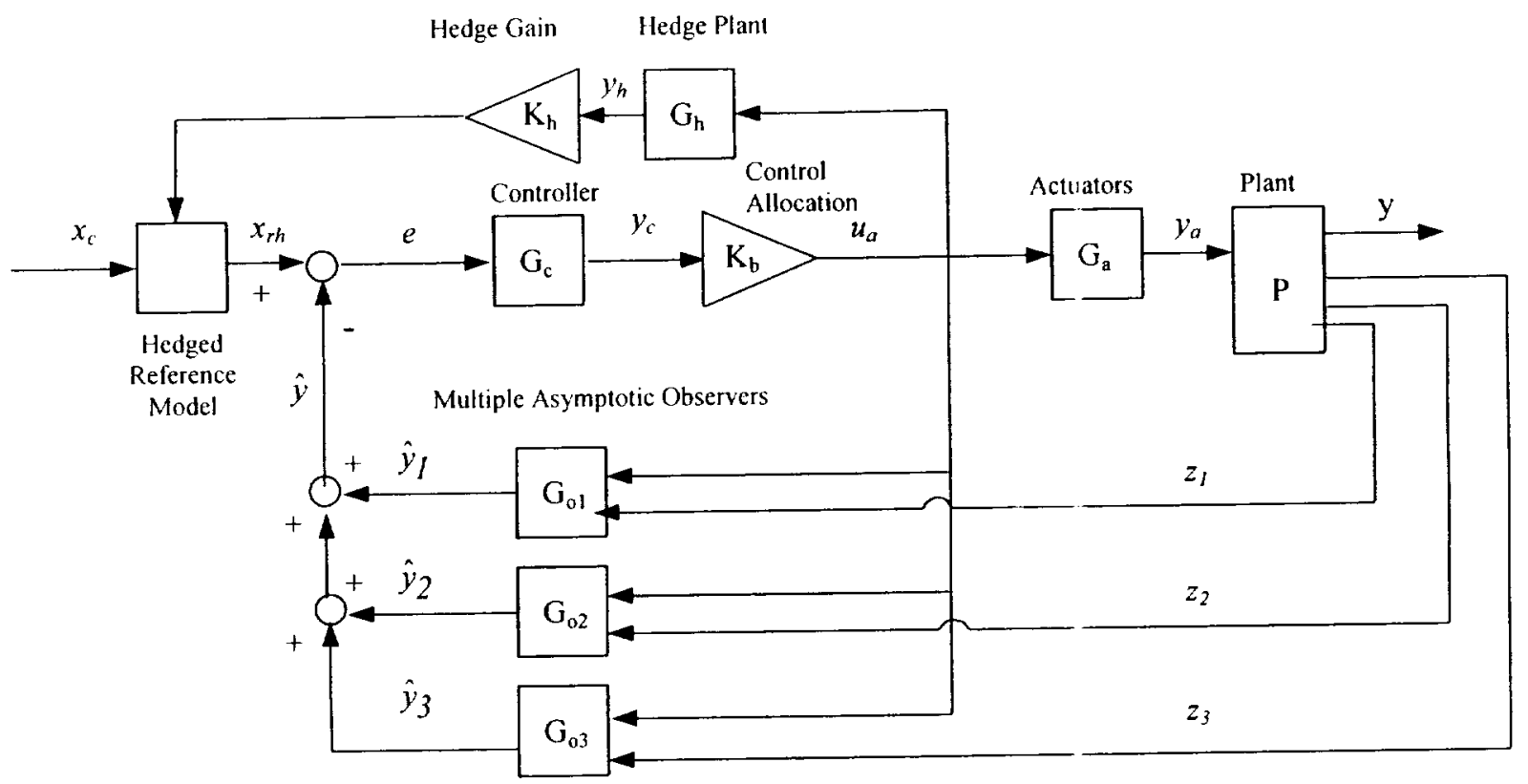

Figure \$: Hedged system architecture 


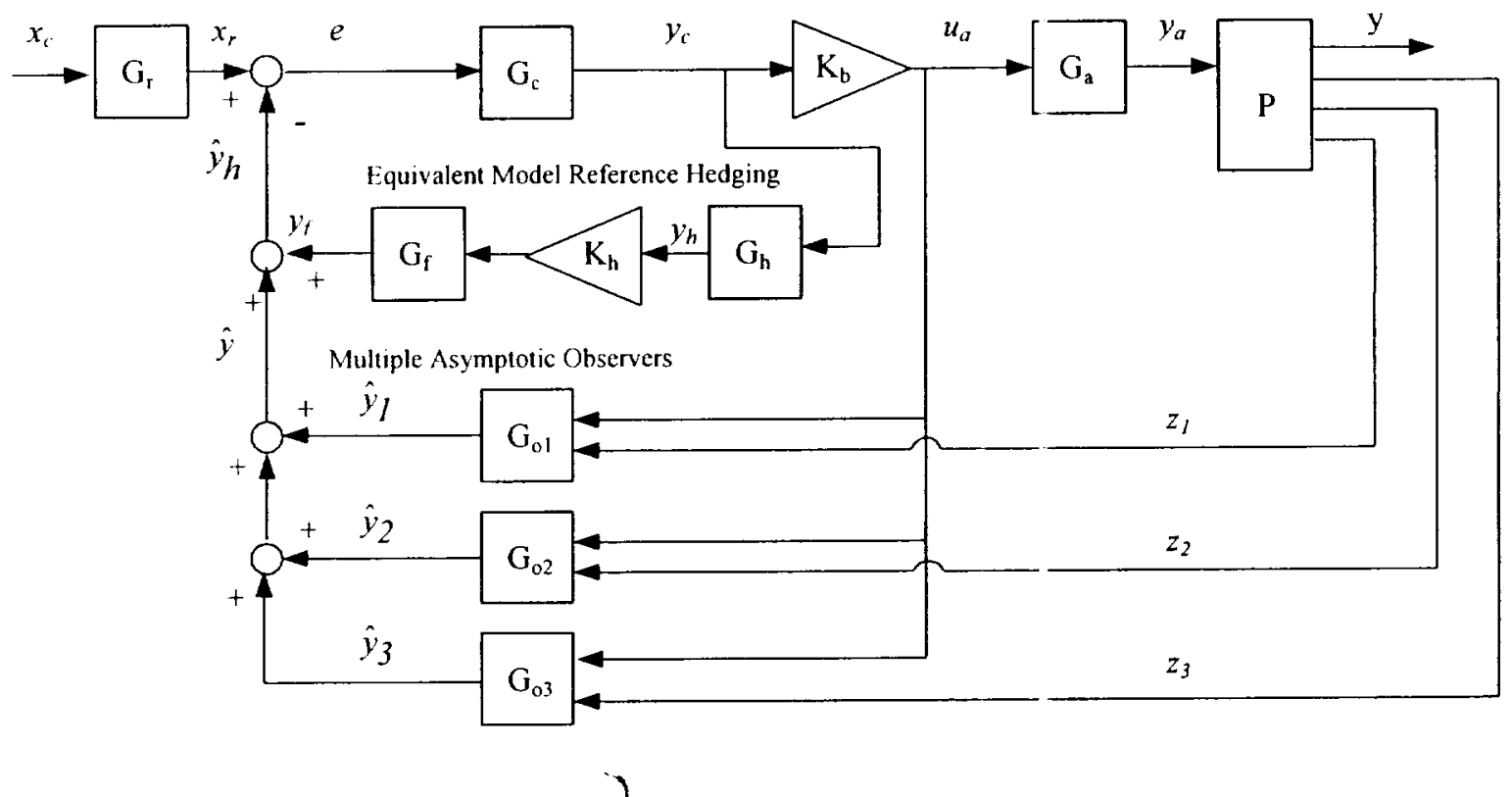

Figure 6: Equivalent hedged system architecture 


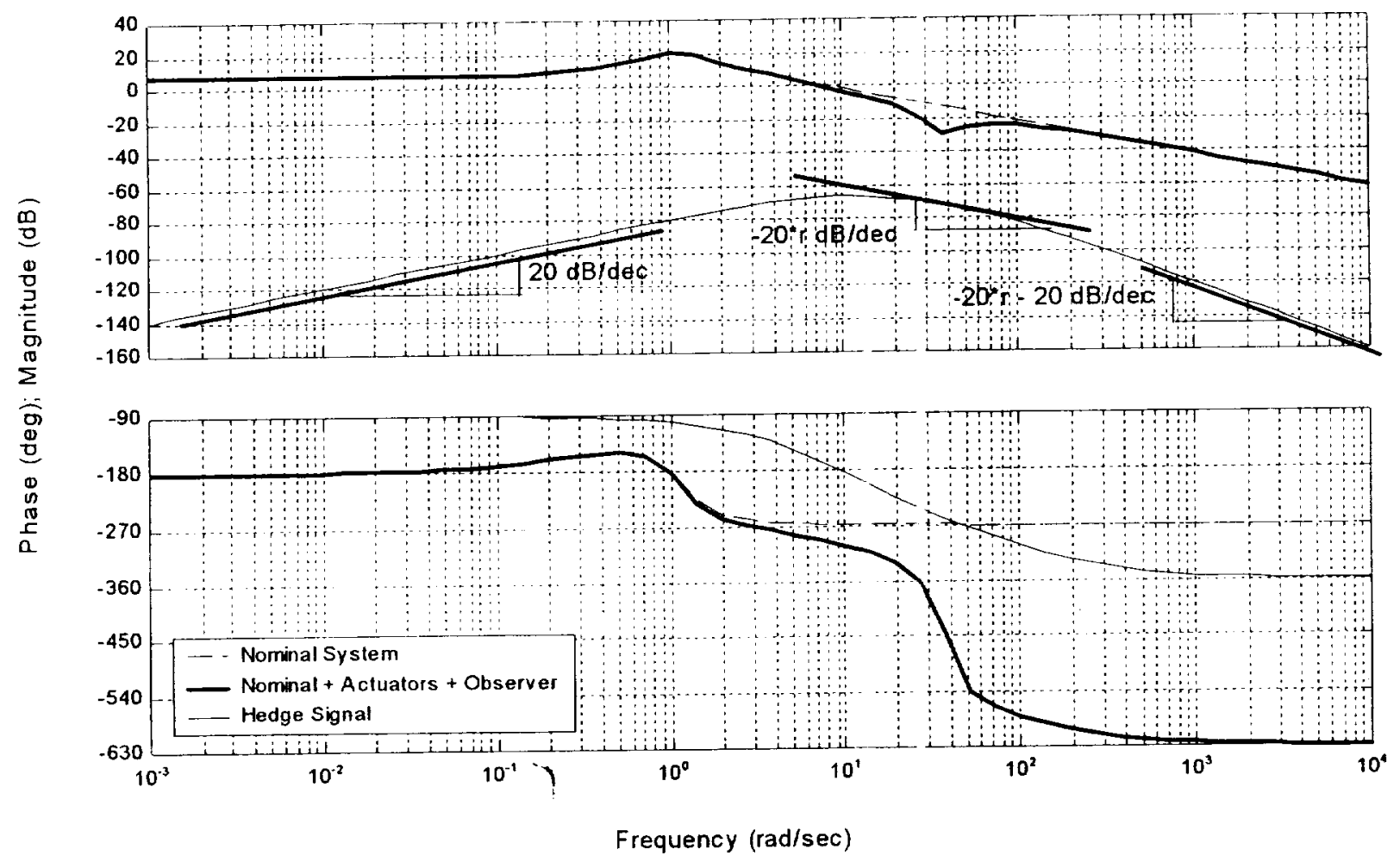

Figure 7: Creating the hedge model, $\frac{\hat{y}_{h}}{y_{c}}$ 


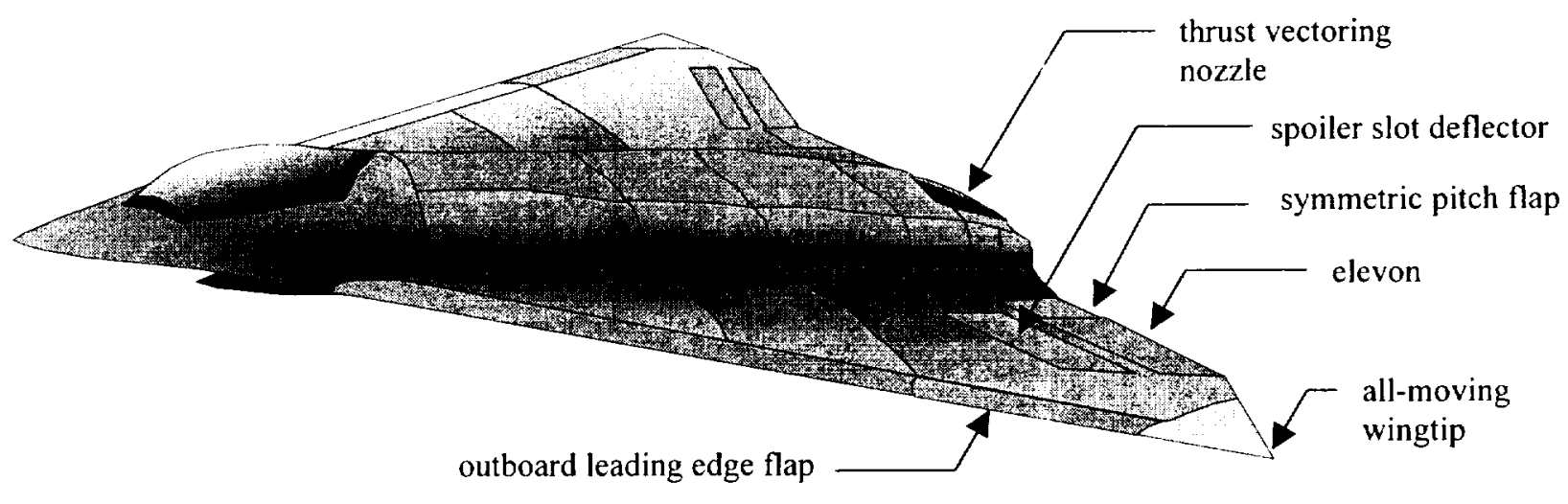

Figure 8: Innovative Control Effector (ICE) vehicle ? 


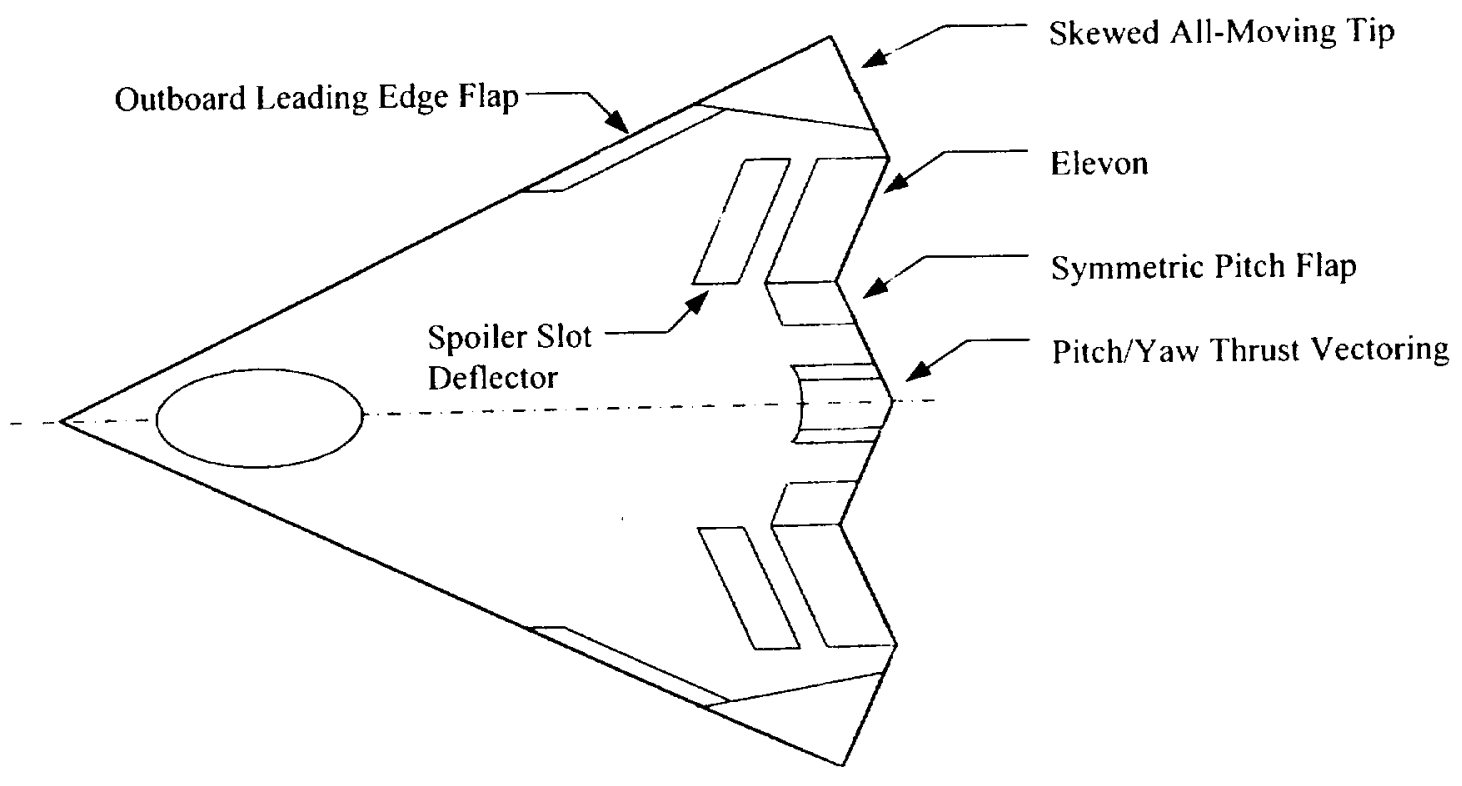

?

Figure 9: ICE control effectors 


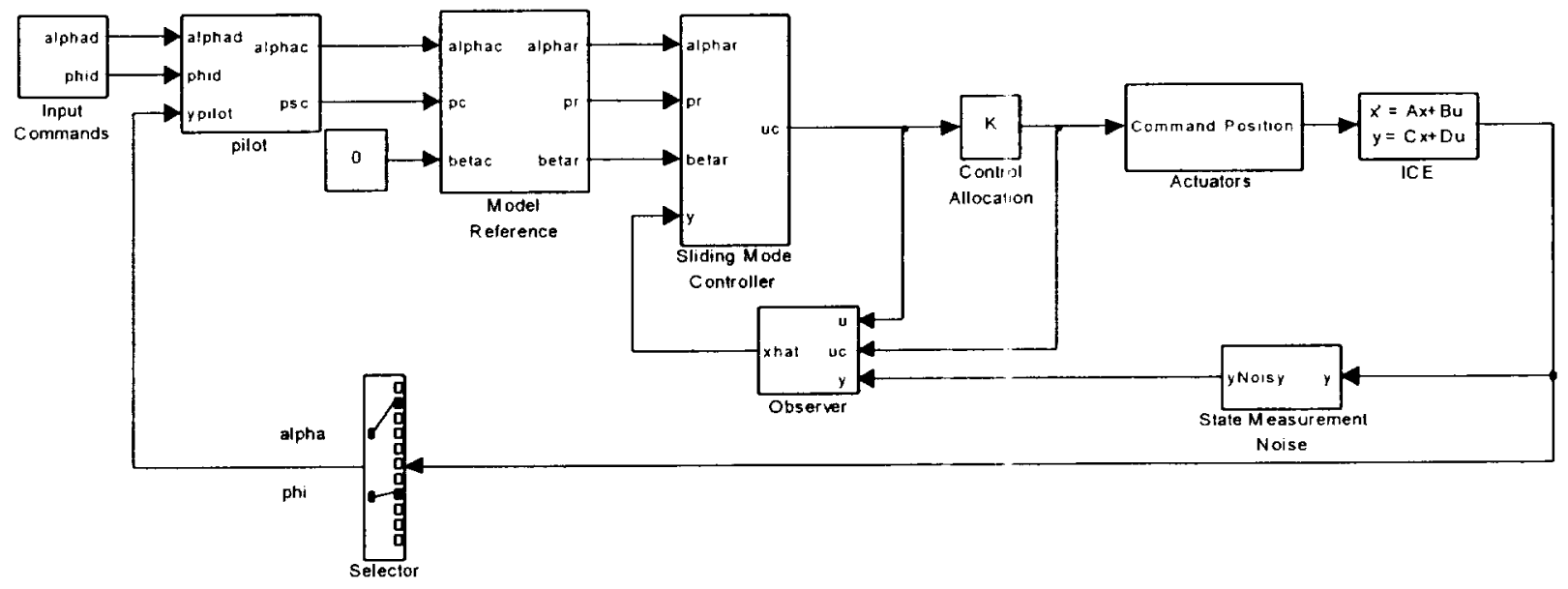

Figure 10: Simulink diagram of the ICE pilot/vehicle/SMC system 

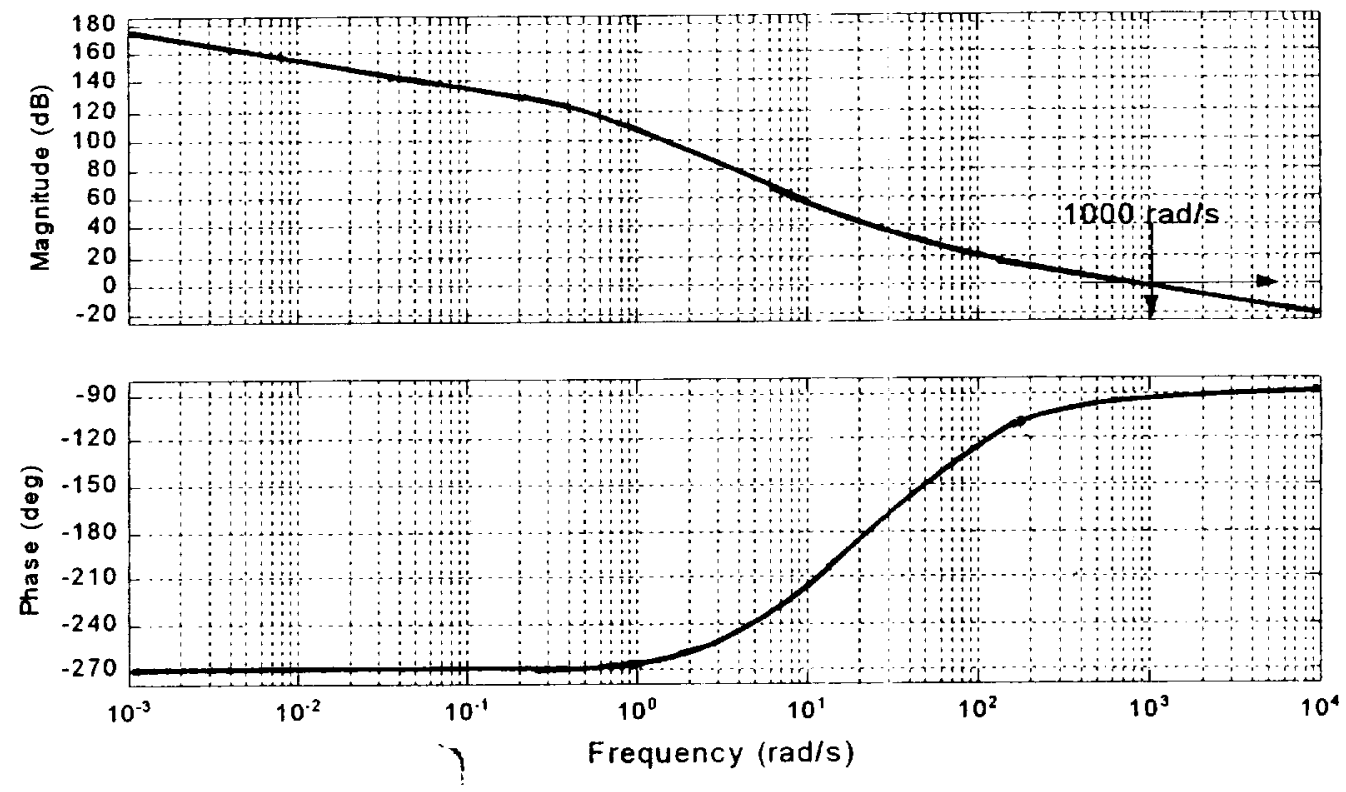

Figure 11: Loop transmission for $\beta$-Loop of Step (4) of ICE SMC design, all other loops closed 

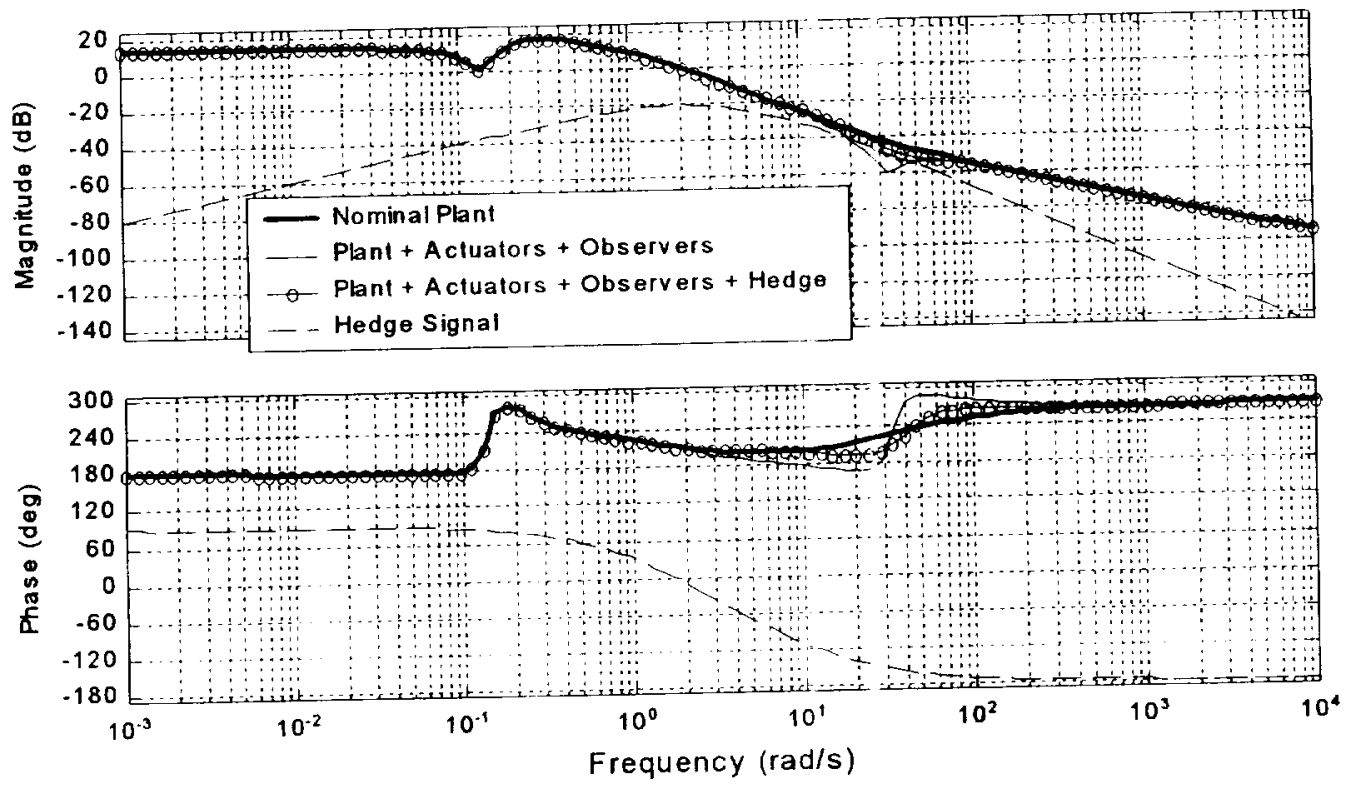

$y$

Figure 12: Bode plots, $\frac{\hat{\alpha}_{\mathrm{h}}}{\mathrm{u}_{\mathrm{c} \alpha}}$, ICE hedge design 

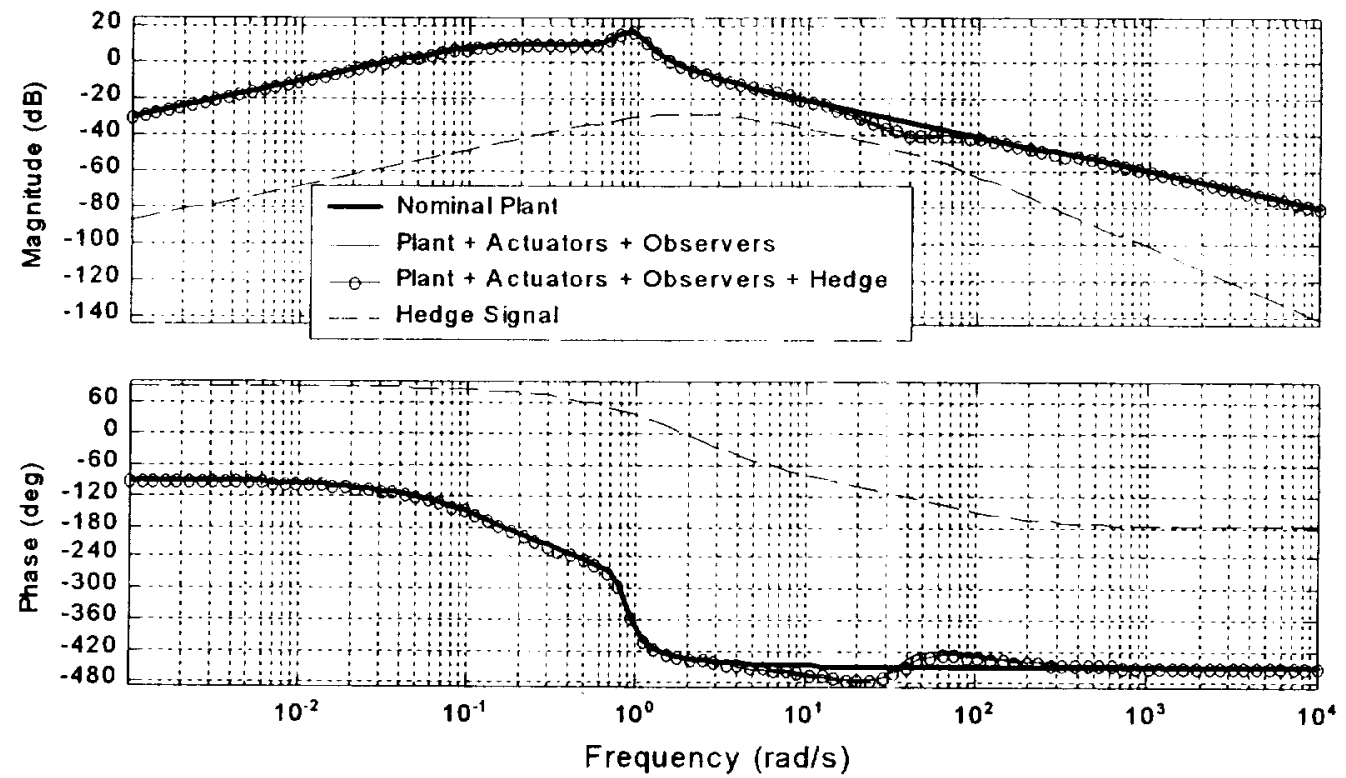

Figure 13: Bode plots, $\frac{\hat{\mathrm{p}}_{\mathrm{h}}}{\mathrm{u}_{\mathrm{cp}}}$, ICE hedge design 

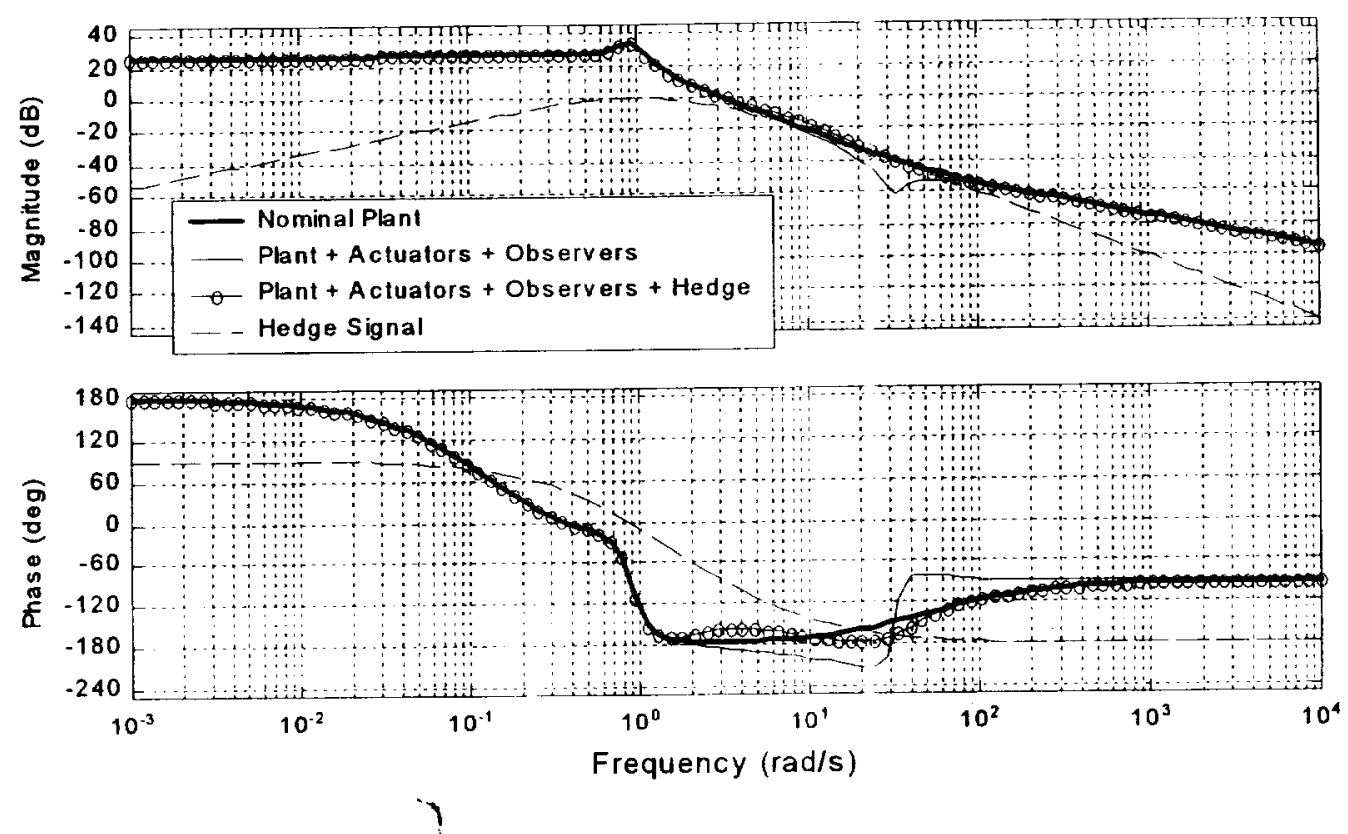

Figure 14: Bode plots, $\frac{\hat{\beta}_{\mathrm{b}}}{\mathrm{u}_{\mathrm{c} \beta}}$, ICE hedge design 


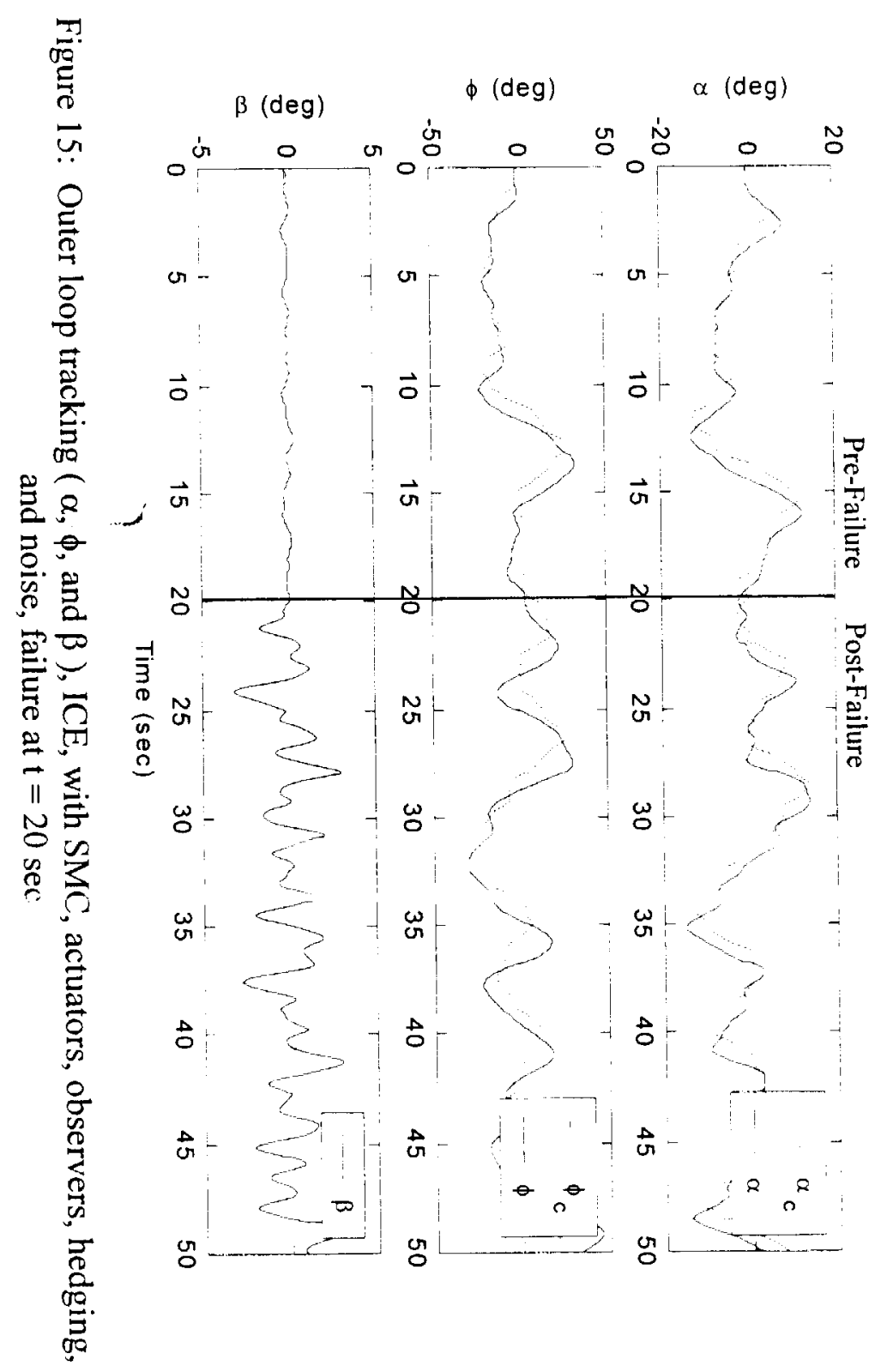



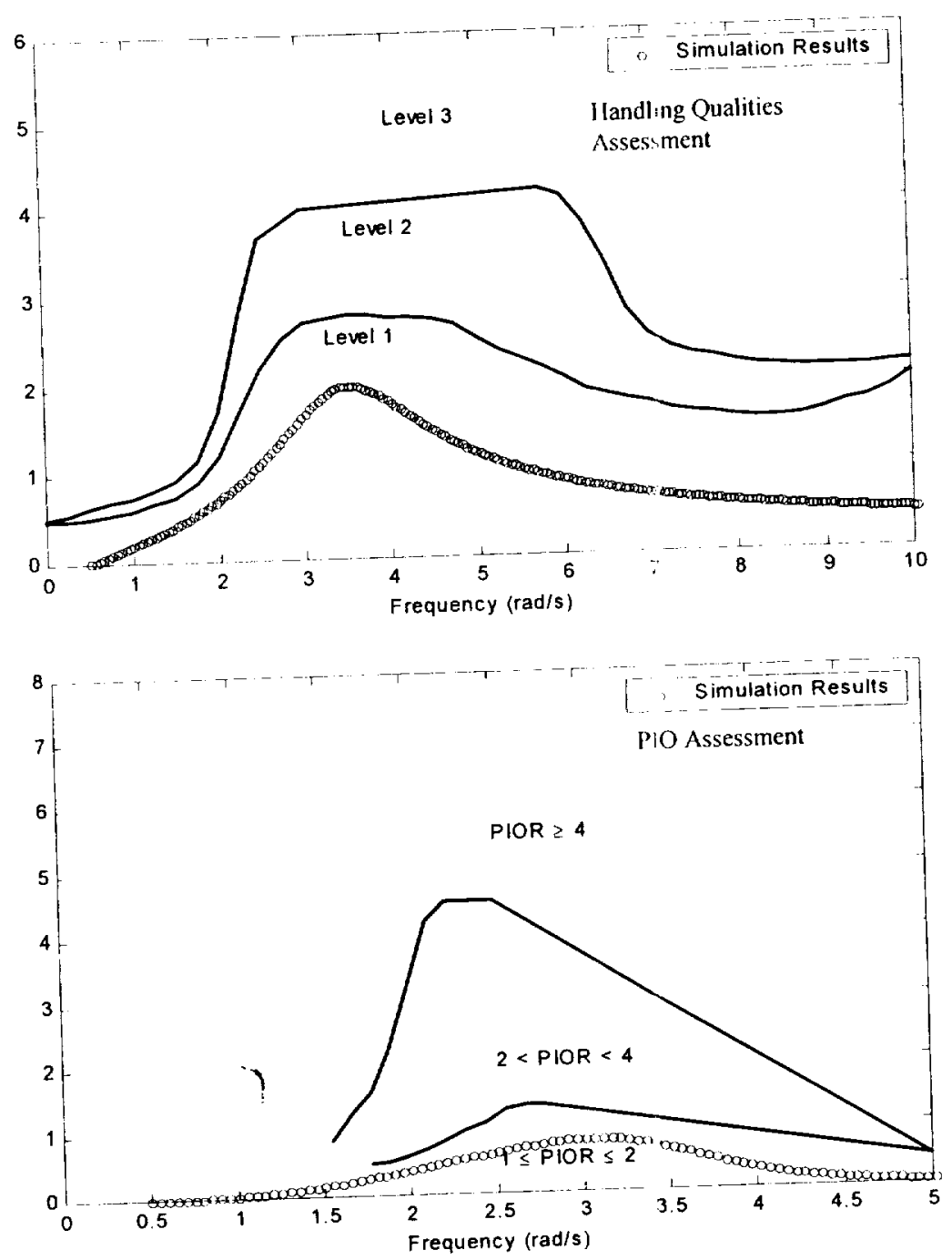

Figure 16: $\alpha$-tracking task HQ and PIO predictions, ICE failed system 


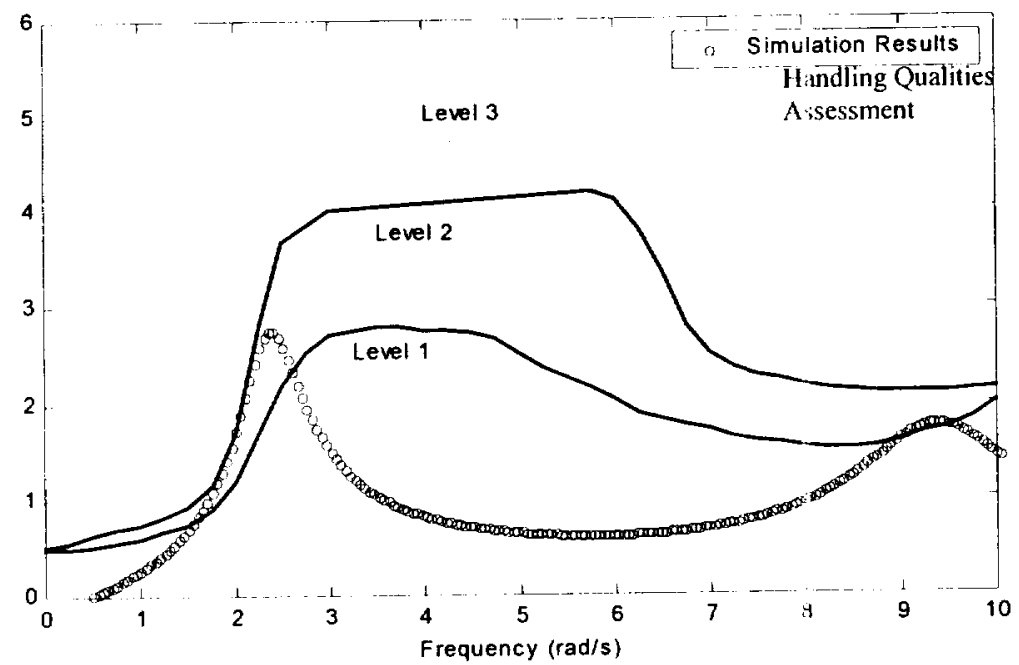

PIO Assessment

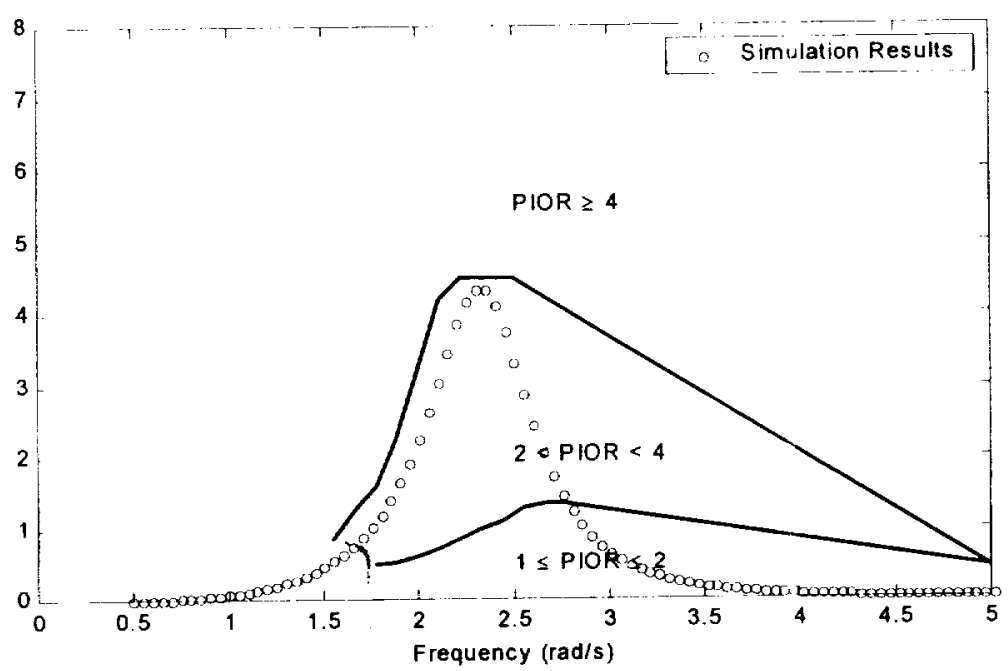

Figure 17: $\phi$-tracking task HQ and PIO predictions, ICE failed system 


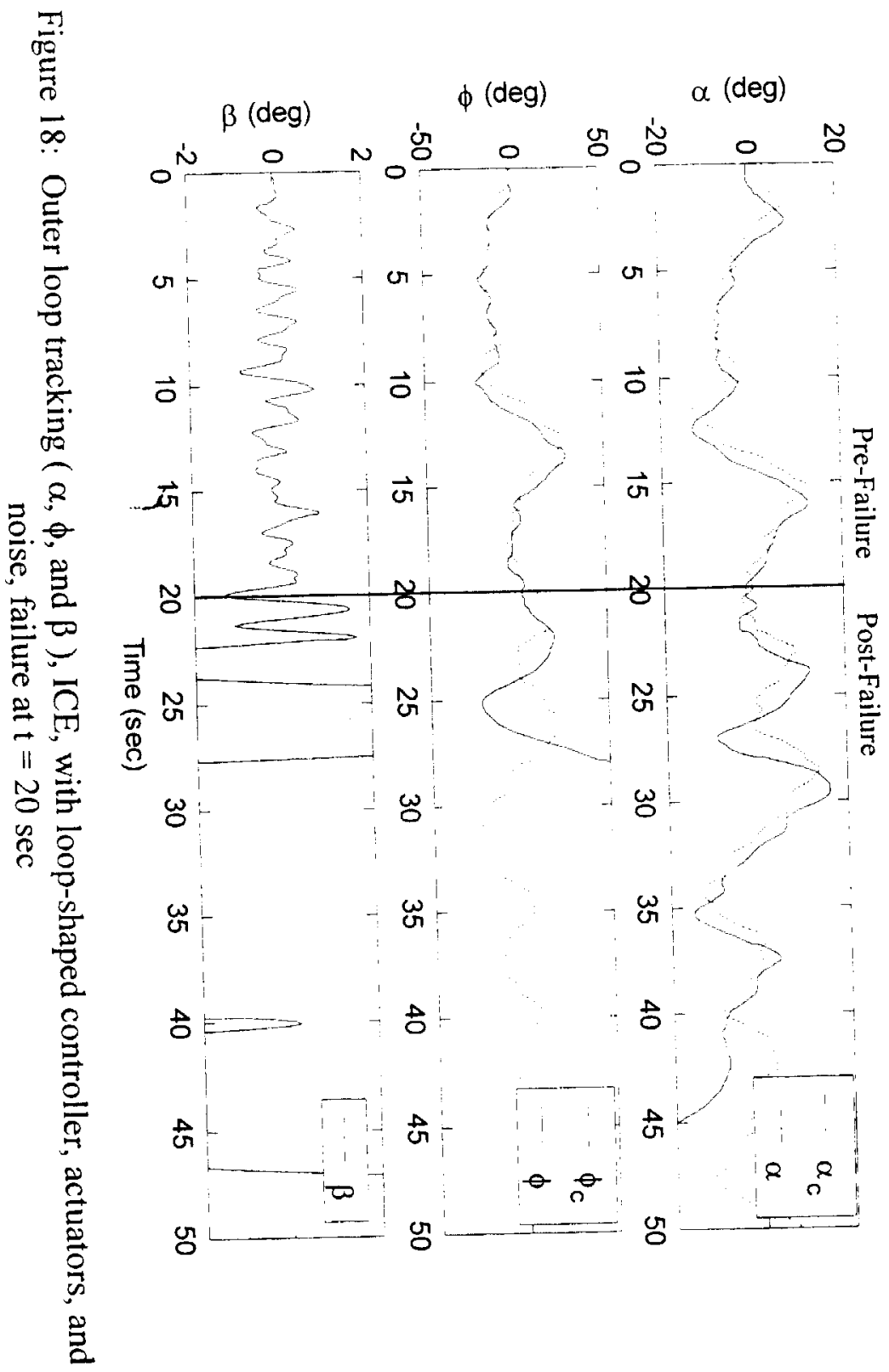

